\title{
SARS-CoV-2 mRNA vaccine design enabled by prototype pathogen preparedness
}

https://doi.org/10.1038/s41586-020-2622-0

Received: 10 June 2020

Accepted: 29 July 2020

Published online: 5 August 2020

Check for updates

\begin{abstract}
Kizzmekia S. Corbett ${ }^{1,0}$, Darin K. Edwards ${ }^{2,10}$, Sarah R. Leist ${ }^{3,10}$, Olubukola M. Abiona', Seyhan Boyoglu-Barnum', Rebecca A. Gillespie', Sunny Himansu' ${ }^{2}$, Alexandra Schäfer ${ }^{3}$, Cynthia T. Ziwawo', Anthony T. DiPiazza', Kenneth H. Dinnon ${ }^{3}$, Sayda M. Elbashir ${ }^{2}$, Christine A. Shaw ${ }^{2}$, Angela Woods ${ }^{2}$, Ethan J. Fritch ${ }^{4}$, David R. Martinez ${ }^{3}$, Kevin W. Bock ${ }^{5}$, Mahnaz Minai ${ }^{5}$, Bianca M. Nagata ${ }^{5}$, Geoffrey B. Hutchinson', Kai Wu ${ }^{2}$, Carole Henry ${ }^{2}$, Kapil Bahl' ${ }^{2}$, Dario Garcia-Dominguez ${ }^{2}$, LingZhi Ma ${ }^{2}$, Isabella Renzi ${ }^{2}$, Wing-Pui Kong', Stephen D. Schmidt', Lingshu Wang', Yi Zhang', Emily Phung 1,6 Lauren A. Chang', Rebecca J. Loomis', Nedim Emil Altaras ${ }^{2}$, Elisabeth Narayanan ${ }^{2}$, Mihir Metkar ${ }^{2}$, Vlad Presnyak' ${ }^{2}$, Cuiping Liu', Mark K. Louder', Wei Shi', Kwanyee Leung', Eun Sung Yang', Ande West ', Kendra L. Gully ${ }^{3}$, Laura J. Stevens ${ }^{7}$, Nianshuang Wang ${ }^{8}$, Daniel Wrapp ${ }^{8}$, Nicole A. Doria-Rose', Guillaume Stewart-Jones ${ }^{2}$, Hamilton Bennett ${ }^{2}$, Gabriela S. Alvarado', Martha C. Nason ${ }^{9}$, Tracy J. Ruckwardt', Jason S. McLellan ${ }^{8}$, Mark R. Denison ${ }^{7}$, James D. Chappell', Ian N. Moore ${ }^{5}$, Kaitlyn M. Morabito', John R. Mascola', Ralph S. Baric ${ }^{3,4}$, Andrea Carfi ${ }^{\circledR} \bowtie$ \& Barney S. Graham ${ }^{1 凶}$
\end{abstract}

\begin{abstract}
A vaccine for severe acute respiratory syndrome coronavirus 2 (SARS-CoV-2) is needed to control the coronavirus disease 2019 (COVID-19) global pandemic. Structural studies have led to the development of mutations that stabilize Betacoronavirus spike proteins in the prefusion state, improving their expression and increasing immunogenicity ${ }^{1}$. This principle has been applied to design mRNA-1273, an mRNA vaccine that encodes a SARS-CoV-2 spike protein that is stabilized in the prefusion conformation. Here we show that mRNA-1273 induces potent neutralizing antibody responses to both wild-type (D614) and D614G mutant ${ }^{2}$ SARS-CoV-2 as well as $\mathrm{CD}^{+} \mathrm{T}$ cell responses, and protects against SARS-CoV-2 infection in the lungs and noses of mice without evidence of immunopathology. mRNA-1273 is currently in a phase III trial to evaluate its efficacy.
\end{abstract}

Since its emergence in December 2019, SARS-CoV-2 has accounted for more than 30 million cases of coronavirus disease 2019 (COVID-19) worldwide in 9 months ${ }^{3}$.SARS-CoV-2 is the third novel Betacoronavirus in the past 20 years to cause substantial human disease; however, unlike its predecessors SARS-CoV and Middle East respiratory syndrome coronavirus (MERS-CoV), SARS-CoV-2 is transmitted efficiently from person to person. In the absence of a vaccine, public health measures such as quarantine of newly diagnosed cases, contact tracing, use of face masks and physical distancing have been put into place to reduce transmission ${ }^{4}$. It is estimated that until $60-70 \%$ of the population have immunity, COVID-19 is unlikely to be sufficiently well-controlled for normal human activities to resume. If immunity remains solely dependent on infection, even at a case fatality rate of $1 \%$, more than 40 million people could succumb to COVID-19 globally ${ }^{5}$. Therefore, rapid development of vaccines against SARS-CoV-2 will be critical for changing the global dynamics of this virus.

The spike (S) protein, a class I fusion glycoprotein analogous to influenza haemagglutinin, respiratory syncytial virus (RSV) fusion glycoprotein (F) and human immunodeficiency virus gp160 (Env), is the major surface protein on the coronavirus virion and the primary target for neutralizing antibodies. S proteins undergo marked structural rearrangement to fuse virus and host cell membranes, enabling delivery of the viral genome into target cells. We previously showed that prefusion-stabilized protein immunogens that preserve neutralization-sensitive epitopes are an effective vaccine strategy for enveloped viruses such as RSV ${ }^{6-10}$. Subsequently, we identified 2 proline substitutions (2P) at the apex of the central helix and heptad repeat 1 that effectively stabilized MERS-CoV, SARS-CoV and human coronavirus HKU1S proteins in the prefusion conformation ${ }^{1,11,12}$. Similar to other prefusion-stabilized fusion proteins, MERS-CoVS(2P) protein was more immunogenic at lower doses than wild-type $S$ protein ${ }^{1}$. The 2P mutation has similar effects on the stability of S proteins from other betacoronaviruses, suggesting a generalizable approach for designing stabilized-prefusion Betacoronavirus S protein antigens for vaccination. Such generalizability is fundamental to the prototype pathogen approach for pandemic preparedness ${ }^{13,14}$.

Coronaviruse have long been predicted to have a high probability of causing zoonotic disease and pandemics ${ }^{15,16}$. As part of our pandemic

${ }^{1}$ Vaccine Research Center, National Institute of Allergy and Infectious Diseases, National Institutes of Health, Bethesda, MD, USA. ${ }^{2}$ Moderna Inc, Cambridge, MA, USA. ${ }^{3}$ Department of Epidemiology, Gillings School of Global Public Health, University of North Carolina at Chapel Hill, Chapel Hill, NC, USA. ${ }^{4}$ Department of Microbiology and Immunology, School of Medicine,

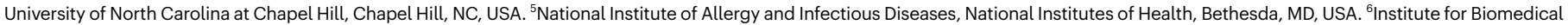
Sciences, George Washington University, Washington, DC, USA. ${ }^{7}$ Department of Pediatrics, Vanderbilt University Medical Center, Nashville, TN, USA. ${ }^{8}$ Department of Molecular Biosciences, University of Texas at Austin, Austin, TX, USA. ${ }^{9}$ Biostatistics Research Branch, Division of Clinical Research, National Institute of Allergy and Infectious Diseases, National Institutes of Health, Bethesda, MD, USA. ${ }^{10}$ These authors contributed equally: Kizzmekia S. Corbett, Darin K. Edwards, Sarah R. Leist. ${ }^{凶}$-mail: andrea.carfi@modernatx.com; bgraham@nih.gov 


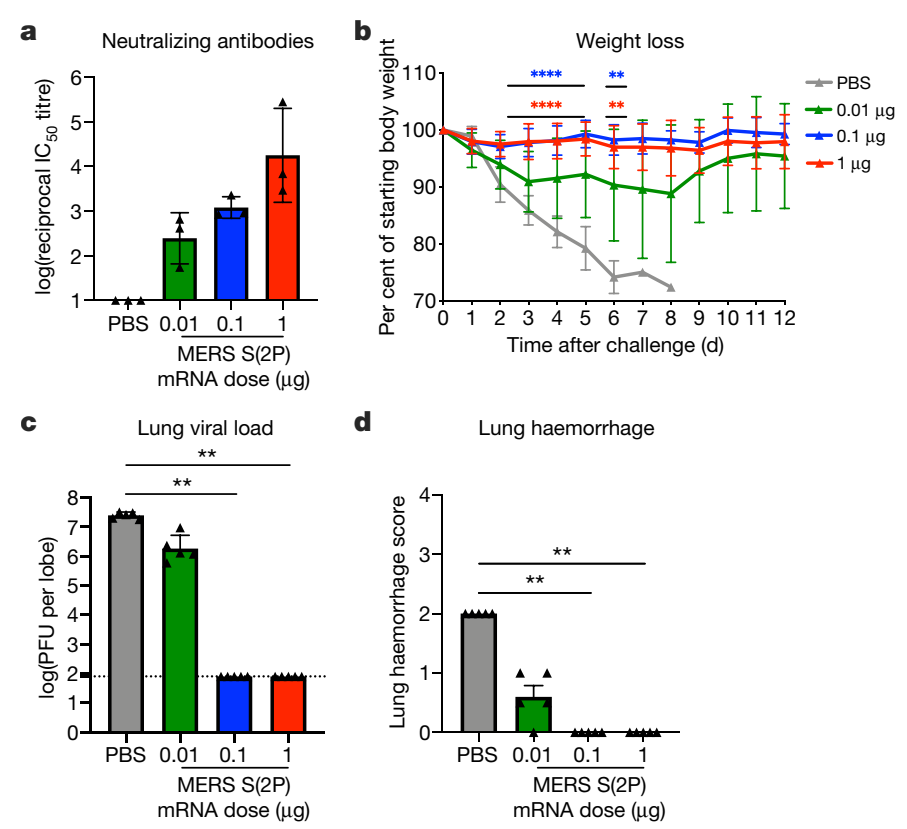

Fig. 1 | MERS-CoV S-2P mRNA protects mice from lethal challenge. a-d, $288 / 330^{+/+}$mice were immunized at weeks 0 and 3 with 0.01 (green), 0.1 (blue) or $1 \mu \mathrm{g}$ (red) MERS-CoV S(2P) mRNA. Control mice were administered phosphate-buffered saline (PBS) (grey). a, Two weeks post-boost, sera were collected from three mice per group and assessed for neutralizing antibodies against MERS m35c4 pseudovirus. b-d, Four weeks post-boost, 12 mice per group were challenged with a lethal dose of mouse-adapted MERS-CoV (m35c4). b, Following challenge, mice were monitored for weight loss. c, d, Two days post-challenge, at peak viral load, lung viral titres (c) and haemorrhage (scored as: 0 , no haemorrhage, 4 , severe haemorrhage in all lobes) (d) were assessed from five mice per group. In c, d, all dose levels were compared by Kruskal-Wallis analysis of variance (ANOVA) with Dunn's multiple comparisons test. In b, for weight loss, all comparisons are with PBS control mice at the same time point by two-sided Mann-Whitney $U$-test. ${ }^{* *} P<0.01,{ }^{* * * *} P<0.0001$. Data are GMT \pm geometric s.d. (a, c) or mean \pm s.d. (b, d). In c, the dotted line represents assay limit of detection.

preparedness efforts, we have studied MERS-CoV as a prototype Betacoronavirus pathogen to optimize vaccine design, dissect the humoral immune response to vaccination, and identify mechanisms and correlates of protection. Achieving an effective and rapid vaccine response to a newly emerging virus requires both the precision afforded by structure-based antigen design and a manufacturing platform to shorten time to product availability. Producing cell lines and clinical-grade subunit protein typically takes more than one year, whereas manufacturing nucleic acid vaccines can be achieved in a matter of weeks ${ }^{17,18}$. In addition to advantages in manufacturing speed, mRNA vaccines are potently immunogenic and elicit both humoral and cellular immunity ${ }^{19-21}$. We therefore evaluated mRNA formulated in lipid nanoparticles (mRNA-LNP) as a delivery vehicle for MERS-CoV $\mathrm{S}(2 \mathrm{P})$, and found that transmembrane-anchored MERS-CoVS (2P) mRNA elicited more potent pseudovirus-neutralizing antibody responses than secreted MERS-CoV S(2P) (Extended Data Fig. 1a). Additionally, consistent with protein immunogens, MERS-CoV S(2P) mRNA was more immunogenic than wild-type MERS-CoV S mRNA (Extended Data Fig. 1b). Immunization with MERS-CoVS(2P) mRNA-LNP elicited potent pseudovirus-neutralizing activity with a dose as low as $0.1 \mu \mathrm{g}$ and protected transgenic mice expressing human $D P P 4\left(288 / 330^{+/+}\right)^{22}$ against lethal MERS-CoV challenge in a dose-dependent manner, establishing that mRNA encoding $\mathrm{S}(2 \mathrm{P})$ protein is protective. Notably, a subprotective $0.01 \mu$ g dose of MERS-CoVS (2P) mRNA did not cause exaggerated disease following MERS-CoV infection, but instead resulted in partial protection against weight loss followed by full recovery without evidence of enhanced illness (Fig. 1).

SARS-CoV-2 was first identified as the cause of an outbreak of respiratory disease in Wuhan, China in early January 2020. Within $24 \mathrm{~h}$ of the release of genomic sequences of SARS-CoV-2 isolates on 10 January 2020 , the $2 \mathrm{P}$ mutations were substituted into $S$ protein residues 986 and 987 to produce prefusion-stabilized SARS-CoV-2 S(2P) protein for structural analysis ${ }^{23}$ and serological assay development ${ }^{24,25}$ in silico, without additional experimental validation. Within 5 days of the release of the sequence, current good manufacturing practice (cGMP) production of mRNA-LNP encoding the SARS-CoV-2S $(2 \mathrm{P})$ as a transmembrane-anchored protein with the native furin cleavage site (mRNA-1273) was initiated in parallel with preclinical evaluation. This led to a first-in-human phase I clinical trial starting on 16 March 2020,66 days after the viral sequence was released, and a phase II trial 74 days later on 29 May 2020 (Extended Data Fig. 2). Expression and antigenicity of the $\mathrm{S}(2 \mathrm{P})$ antigen delivered by mRNA was confirmed in vitro before vaccination of the first human participant (Extended Data Fig. 3), and immunogenicity of mRNA-1273 was documented in several mouse strains. The results of those studies are detailed here. a

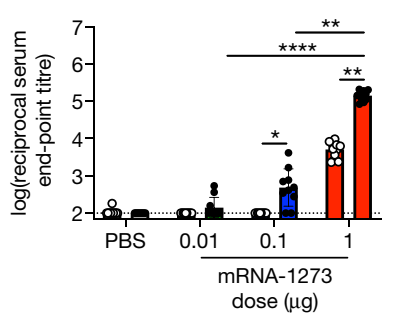

d

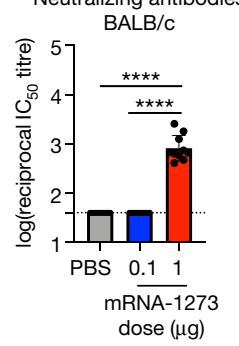

b

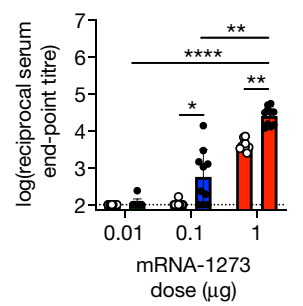

e

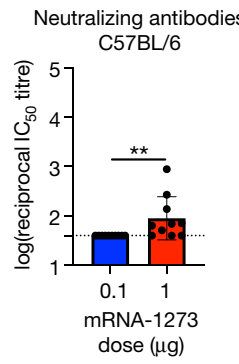

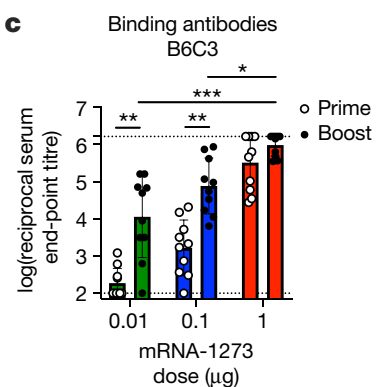

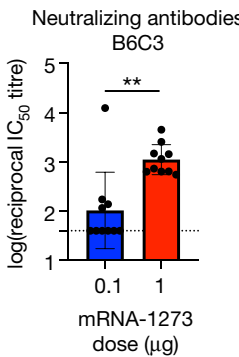

Fig. 2 | mRNA-1273 elicits robust binding and pseudovirus-neutralizing antibody responses in multiple mouse strains. a-f, BALB/cJ (a, d), C57BL/6J (b, e) or B6C3F1/J (c,f) mice ( $n=10$ per group) were immunized at weeks 0 and 3 with 0.01 (green), 0.1 (blue) or $1 \mu \mathrm{g}$ (red) mRNA-1273. ControlBALB/cJ mice were administered PBS (grey). Sera were collected 2 weeks post-prime (unfilled circles) and 2 weeks post-boost (filled circles) and assessed for SARS-CoV-2 S-specific IgG by enzyme-linked immunosorbent assay (ELISA) (a-c), and for post-boost sera, neutralizing antibodies against homotypic SARS-CoV-2 pseudovirus (d-f). In a-c, time points were compared within each dose level by two-sided Wilcoxon signed-rank test, and doses were compared post-boost by Kruskal-Wallis ANOVA with Dunn's multiple comparisons test. In d-f, Vaccine groups were compared by two-sided Mann-Whitney $U$-test. ${ }^{*} P<0.05,{ }^{* *} P<0.01,{ }^{* * *} P<0.001,{ }^{* * * *} P<0.0001$. Data are presented as GMT \pm geometric s.d. Dotted lines represent assay limits of detection. 


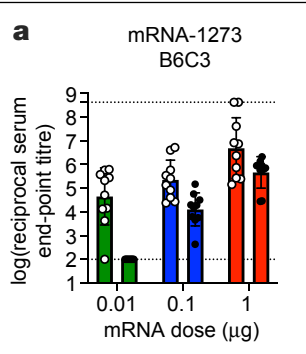

b SARS-CoV-2
S6C3 $(2 \mathrm{P})$ protein $+\mathrm{SAS}$

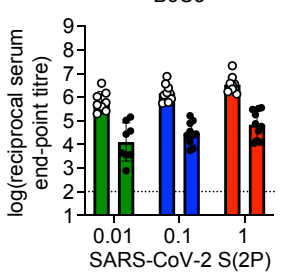

dose $(\mu \mathrm{g})$

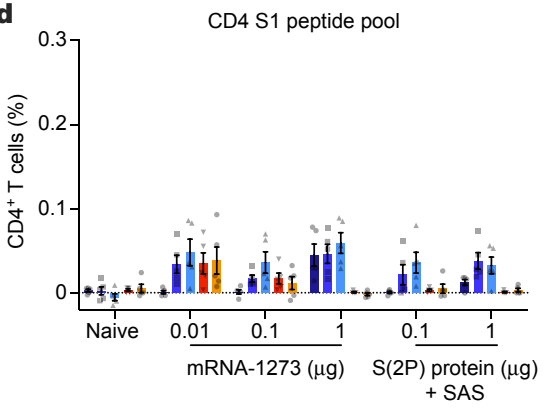

$\mathbf{f}$

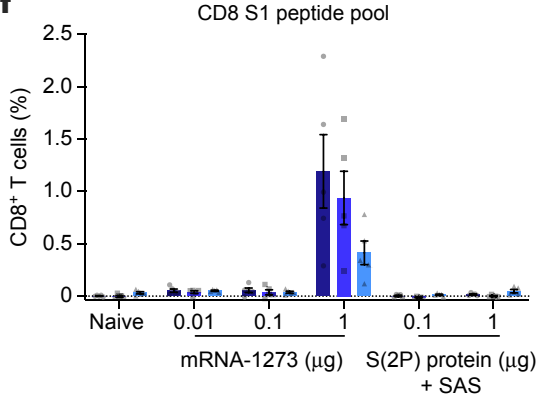

e

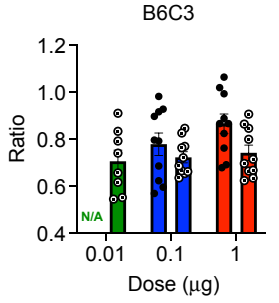

C $(\lg G 2 a+\lg G 2 c) / \lg G 1$ ratio
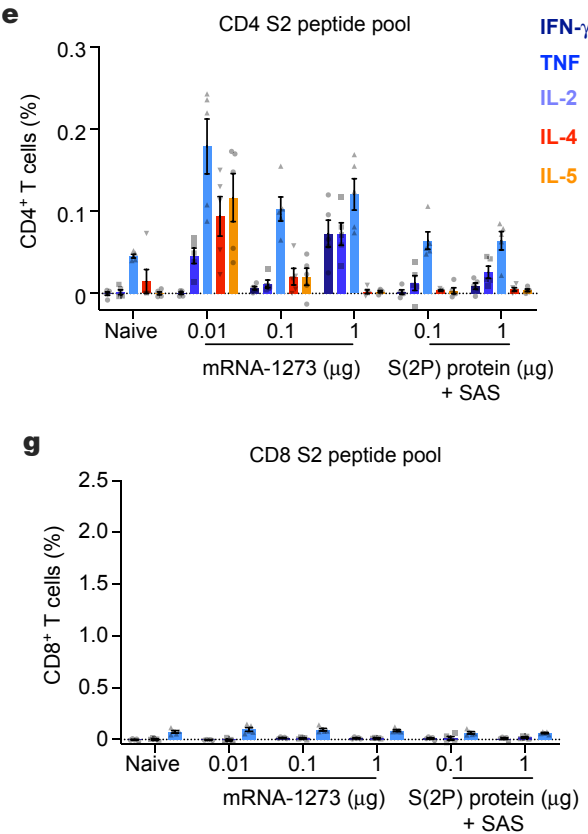

Fig. 3 | Immunizations with mRNA-1273 and S(2P) protein, delivered with TLR4 agonist, elicit $S$-specific $T_{H} 1$-biased $T$ cell responses. $B 6 C 3 F 1 / J$ mice ( $n=10$ per group) were immunized at weeks 0 and 3 with $0.01,0.1$ or $1 \mu \mathrm{g}$ of mRNA-1273 or SARS-CoV-2S(2P) protein with SAS adjuvant. a-c, Sera were collected two weeks post-boost and assessed by ELISA for SARS-CoV-2 S-specific IgG1, and IgG2a and IgG2c. End-point titres $(\mathbf{a}, \mathbf{b})$ and end-point titre ratios of IgG2a plus IgG2c to IgG1 (c) were calculated. Ratios were not calculated for mice for which end-point titres did not reach the lower limit of detection (dotted line; N/A). d-g, Seven weeks post-boost, splenocytes were isolated from five mice per group and restimulated with vehicle or pools of

Immunogenicity was assessed in six-week-old female BALB/cJ, $\mathrm{C} 57 \mathrm{BL} / 6 \mathrm{~J}$ and $\mathrm{B} 6 \mathrm{C} 3 \mathrm{~F} 1 / \mathrm{J}$ mice by two intramuscular immunizations with $0.01,0.1$ or $1 \mu \mathrm{g}$ mRNA-1273, separated by a 3-week interval. mRNA-1273 induced dose-dependent specific S-binding antibodies after prime and boost in all mouse strains (Fig. 2a-c). Potent pseudovirus-neutralizing activity was elicited by $1 \mu \mathrm{g}$ mRNA-1273, reaching reciprocal half-maximal inhibitory concentration $\left(\mathrm{IC}_{50}\right)$ geometric mean titres (GMTs) of $819(\mathrm{BALB} / \mathrm{cJ}), 89(\mathrm{C} 57 \mathrm{BL} / 6 \mathrm{~J})$ and 1,115 (B6C3F1/J) (Fig. 2d-f). Additionally, mice immunized with $1 \mu \mathrm{g}$ mRNA-1273 had robust neutralizing antibodies against pseudoviruses that express S protein with the D614G substitution; SARS-CoV-2 expressing the D614G variant of the S protein has recently become dominant around the world ${ }^{2}$ (Extended Data Fig. 4). To further gauge immunogenicity across a wide dose range, BALB/c mice were immunized with $0.0025-20 \mu \mathrm{g}$ mRNA-1273, revealing a strong positive correlation between dose-dependent mRNA-1273-elicited binding and pseudovirus-neutralizing antibody responses (Extended Data Fig. 5). $\mathrm{BALB} / \mathrm{cJ}$ mice that received a single dose of mRNA-1273 were evaluated to ascertain the utility of a single-dose vaccine. S-binding antibodies were induced in mice immunized with one $1 \mu \mathrm{g}$ or $10 \mu \mathrm{g}$ dose of mRNA-1273. The $10 \mu$ g dose elicited pseudovirus-neutralizing antibody overlapping peptides from SARS-CoV-2 S protein in the presence of a protein transport inhibitor cocktail. After $6 \mathrm{~h}$, intracellular cytokine staining was performed to quantify $\mathrm{CD}^{+}$and $\mathrm{CD}^{+} \mathrm{T}$ cell responses. Cytokine expression in the presence of vehicle only was considered as background and subtracted from the responses measured from the S1 and S2 peptide pools for each individual mouse. d, e, Percentage of $\mathrm{CD}^{+}{ }^{+} \mathrm{T}$ cells expressing IFN- $\gamma, \mathrm{TNF}$, IL-2, IL-4 and IL-5 in response to the S1 (d) and S2 (e) peptide pools.f, g, Percentage of $\mathrm{CD}^{+} \mathrm{T}$ cells expressing IFN- $\gamma, \mathrm{TNF}$ and IL-2 in response to the S1 (f) and S2 (g) peptide pools.

activity that increased between week 2 and week 4 , reaching 315 reciprocal IC ${ }_{50}$ GMT (Extended Data Fig. 6a, b). These data demonstrate that mRNA expressing SARS-CoV-2 S $(2 \mathrm{P})$ is a potent immunogen and that pseudovirus-neutralizing activity can be elicited with a single dose.

Vaccine-associated enhanced respiratory disease (VAERD) has been associated with $T$ helper 2 cell $\left(\mathrm{T}_{\mathrm{H}} 2\right)$-biased immune responses in children immunized with whole-inactivated-virus vaccines against RSV and measles virus ${ }^{26,27}$. A similar phenomenon has also been reported in some animal models with whole-inactivated vaccines and other types of experimental SARS-CoV vaccines ${ }^{28-30}$. We therefore evaluated the balance of $\mathrm{T}_{\mathrm{H}} 1$ and $\mathrm{T}_{\mathrm{H}} 2$ cells in immunized mice. We first compared levels of S-specific immunoglobulins, IgG2a and IgG2c, and IgG1which are surrogates of $\mathrm{T}_{\mathrm{H}} 1$ and $\mathrm{T}_{\mathrm{H}} 2$ responses, respectively-elicited by mRNA-1273 with those elicited by immunization with SARS-CoV-2 $\mathrm{S}(2 \mathrm{P})$ protein using the TLR4 agonist Sigma Adjuvant System (SAS). Both immunogens elicited S-binding antibodies in the IgG2a and IgG1 subclasses, indicating a balanced $\mathrm{T}_{\mathrm{H}} 1-\mathrm{T}_{\mathrm{H}} 2$ response (Fig. $3 \mathrm{a}-\mathrm{c}$, Extended Data Fig. 7). The S-specific IgG-subclass profile following a single dose of mRNA-1273 (Extended Data Fig. 6c) was similar to that observed following two doses. By contrast, $\mathrm{T}_{\mathrm{H}} 2$-biased responses, with lower IgG2a/IgG1 ratios, were observed in mice immunized with 


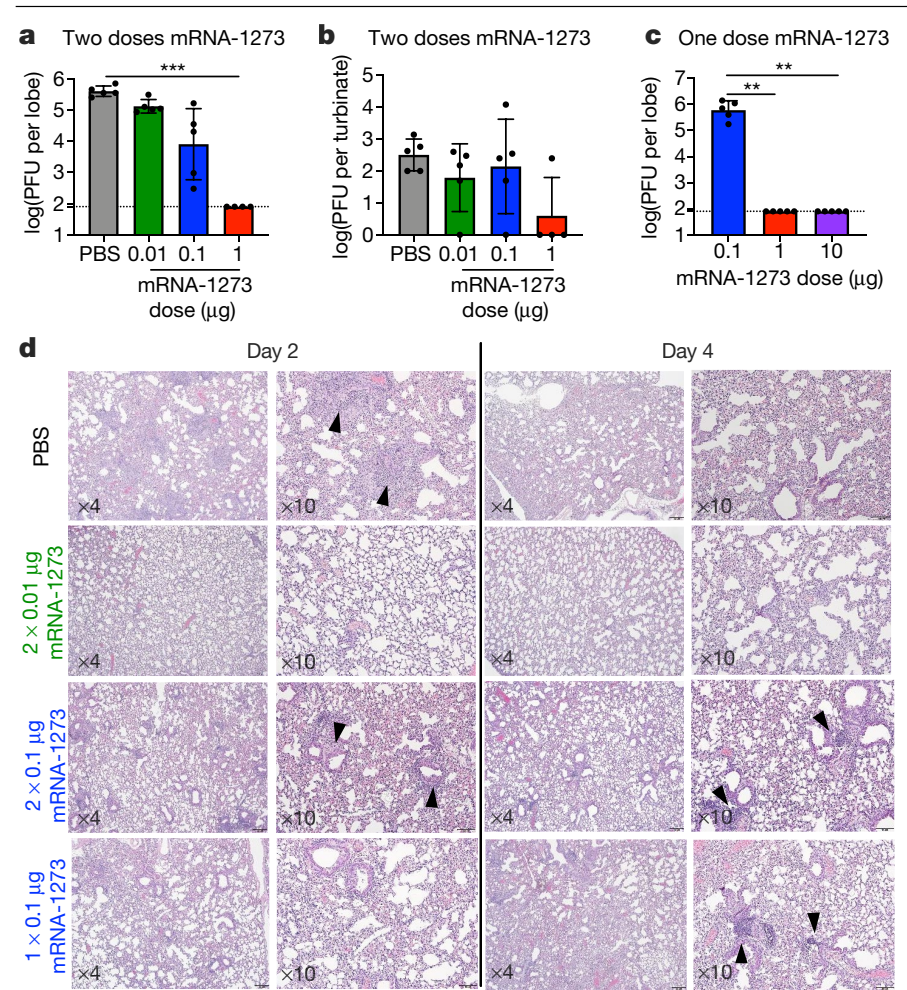

Fig. 4 | mRNA-1273 protects mice from upper-and lower-airway SARS-CoV-2 infection. a, b, BALB/cJ mice ( $n=10$ per group) immunized at weeks 0 and 3 with $0.01 \mu \mathrm{g}$ (green), $0.1 \mu \mathrm{g}$ (blue) or $1 \mu \mathrm{g}$ (red) mRNA-1273 or PBS were challenged with SARS-CoV-2 MA five weeks post-boost. c, Other groups were immunized with single doses of $0.1 \mu \mathrm{g}$ (blue), $1 \mu \mathrm{g}$ (red) or $10 \mu \mathrm{g}$ (purple) mRNA-1273 and challenged 7 weeks after immunization. Two days after challenge, at peak viral load, mouse lungs (a, c) and nasal turbinates (b) were collected from five mice per group to measure viral titres. $\mathbf{a}-\mathbf{c}$, Data are presented as GMT \pm geometric s.d. and dotted lines represent assay limits of detection. Group comparisons were made by Kruskal-Wallis ANOVA with Dunn's multiple comparisons test. ${ }^{* *} P<0.01,{ }^{* * *} P<0.001$. d, At days 2 and 4 after challenge, lung sections from 5 mice per group were stained with haematoxylin and eosin, and representative photomicrographs (original magnification $\times 4$ (scale bars, $600 \mu \mathrm{m})$ and $\times 10$ (scale bars, $300 \mu \mathrm{m})$ as indicated) from each group with detectable virus in lung are shown. Day 2 lungs from PBS control mice demonstrated moderate-to-severe, predominantly neutrophilic inflammation present within and surrounding small bronchioles (arrowheads); alveolar capillaries were markedly expanded by infiltrating inflammatory cells. In the $0.01 \mu \mathrm{g}$ two-dose group, inflammation was minimal to absent. In the $0.1 \mu \mathrm{g}$ two-dose group, occasional areas of inflammation intimately associated with small airways (bronchioles) and adjacent vasculature (arrowheads) were seen, primarily composed of neutrophils. In the single-dose $0.1 \mu$ g group, there were mild patchy expansions of alveolar septae by mononuclear and polymorphonuclear cells. At day 4 , lungs from PBS control mice exhibited moderate-to-marked expansion of alveolar septae (interstitial pattern) with decreased prominence of adjacent alveolar spaces. In the $0.01 \mu \mathrm{g}$ two-dose group, inflammation was minimal to absent. Lungs in the $0.1 \mu \mathrm{g}$ two-dose group showed mild, predominantly lymphocytic inflammation, associated with bronchioles and adjacent vasculature (arrowheads). In the single-dose $0.1 \mu$ g group there was mild, predominantly lymphocytic inflammation around bronchovascular bundles (arrowheads).

SARS-CoV-2S(2P) protein formulated in alum (Extended Data Fig. 8a, b). Following restimulation with peptide pools (one pool of overlapping peptides for each S subunit, S1 and S2) covering the entire S protein, splenocytes from mice immunized with mRNA-1273 secreted more IFN- $\gamma$ (a prototypic $\mathrm{T}_{\mathrm{H}} 1$ cytokine) than IL-4, IL-5 or IL-13 (classical $\mathrm{T}_{\mathrm{H}} 2$ cytokines), whereas restimulation with SARS-CoV-2S(2P) protein with alum adjuvant induced a $\mathrm{T}_{\mathrm{H}} 2$-biased response (Extended Data Fig. $8 \mathrm{c}, \mathrm{d}$ ).
We also directly measured cytokine patterns in vaccine-induced memory T cells by intracellular cytokine staining seven weeks after the boost injection; mRNA-1273-elicited $\mathrm{CD}^{+}{ }^{+} \mathrm{T}$ cells re-stimulated with $\mathrm{S} 1$ or S2 peptide pools exhibited a $\mathrm{T}_{\mathrm{H}} 1$-dominant response, particularly at higher immunogen doses (Fig. 3d, e). Furthermore, $1 \mu \mathrm{g}$ mRNA-1273 induced a robust $\mathrm{CD} 8^{+} \mathrm{T}$ cell response to the $\mathrm{S1}$ peptide pool (Fig. $3 \mathrm{f}$, g). Together, the IgG subclass and $T$ cell cytokine data demonstrate that immunization with mRNA-1273 elicits balanced $\mathrm{T}_{\mathrm{H}} 1$ and $\mathrm{T}_{\mathrm{H}} 2$ responses, in contrast to the $T_{\mathrm{H}} 2$-biased response seen when using $S$ protein with alum adjuvant, suggesting that $\mathrm{mRNA}$ vaccination avoids $\mathrm{T}_{\mathrm{H}} 2$-biased immune responses, which have been linked to VAERD.

Protective immunity was assessed in young adult BALB/cJ mice challenged with mouse-adapted (MA) SARS-CoV-2. SARS-CoV-2 MA contains the substitutions Q498Y/P499T in the receptor-binding domain $^{31}$. The substitutions enable the virus to bind to the mouse angiotensin-converting enzyme 2 (ACE2) receptor and infect and replicate in the upper and lower respiratory $\operatorname{tract}^{32}$. BALB/cJ mice that received two $1 \mu \mathrm{g}$ doses of $\mathrm{mRNA}-1273$ were completely protected from viral replication in lungs after challenge 5 or 13 weeks after boost injection (Fig. 4a, Extended Data Fig. 9a). mRNA-1273-induced immunity also resulted in undetectable viral replication in nasal turbinates in 6 out of 7 mice (Fig. 4b, Extended Data Fig. 9b). The efficacy of mRNA-1273 was dose-dependent; two $0.1 \mu \mathrm{g}$ doses of mRNA-1273 reduced lung viral load by about 100 -fold, whereas two $0.01 \mu \mathrm{g}$ doses reduced lung viral load by about 3 -fold (Fig. 4a). Of note, mice challenged 7 weeks after a single dose of 1 or $10 \mu \mathrm{g}$ mRNA-1273 were also completely protected against lung viral replication (Fig. 4c). Challenging animals immunized with subprotective doses provides an orthogonal assessment of safety signals such as increased clinical illness or pathology. Similar observations with MERS-CoVS(2P) mRNA, mice immunized with subprotective 0.1 or $0.01 \mu \mathrm{g}$ doses of mRNA-1273 showed no evidence of enhanced lung pathology or excessive mucus production (Fig. 4d). In summary, mRNA-1273 is immunogenic, efficacious and does not produce evidence of VAERD when given at subprotective doses in mice.

Here we have shown that $1 \mu \mathrm{g}$ of mRNA-1273 is sufficient to induce robust pseudovirus-neutralizing activity and CD8 T cell responses, balanced $\mathrm{T}_{\mathrm{H}} 1-\mathrm{T}_{\mathrm{H}} 2$ antibody isotype responses, and protection from viral replication for more than three months following a primeboost regimen similar to the one being tested in humans. The level of pseudovirus-neutralizing activity induced by $1 \mu \mathrm{g}$ mRNA-1273 in mice is similar in magnitude to that induced by $100 \mu \mathrm{g}$ mRNA-1273 in humans ${ }^{33}$, which is the dose selected for mRNA-1273 to advance into phase III clinical trials. The inclusion of lower subprotective doses demonstrates the dose-dependence of antibody, $\mathrm{T}_{\mathrm{H}} 1 \mathrm{CD} 4 \mathrm{~T}$ cell responses and protection, suggesting that immune correlates of protection can be further elucidated. Animal studies supporting candidate SARS-CoV-2 vaccines through clinical trials aim to demonstrate elicitation of potent protective immune responses as well as to show that subprotective responses do not cause VAERD ${ }^{5}$. Subprotective doses of mRNA-1273 did not prime mice for enhanced immunopathology following challenge. Moreover, the induction of protective immunity following a single dose suggests single-dose administration of this vaccine could be considered in the outbreak setting. These data, combined with immunogenicity data from non-human primates and human participants of early phase I clinical trials, have been used to inform the dose and regimen of mRNA1273 in advanced clinical efficacy trials.

The COVID-19 pandemic of 2020 is the widely predicted 'pathogen X event ${ }^{\prime 33,14}$. Here we provide a paradigm for rapid vaccine development. Combining structure-guided stabilization of the MERS-CoVS protein with a fast, scalable and safe mRNA-LNP vaccine platform has led to a generalizable vaccine solution for Betacoronavirus and a commercial mRNA vaccine delivery platform; these developments enabled a rapid response to the COVID-19 outbreak. This response demonstrates how new technology-driven concepts such as synthetic vaccinology can facilitate a vaccine development programme initiated on the basis of 
pathogen sequences alone ${ }^{11}$. This study also provides a proof of concept for the prototype-pathogen approach to pandemic preparedness and response that is predicated on identifying generalizable solutions for medical counter measures within virus families or genera ${ }^{13}$. Although the response to the COVID-19 pandemic has been unprecedented in its speed and breadth, we envision further improvements in rapid responses to such threats. There are 24 other virus families that are known to infect humans, and sustained investigation of those potential threats will improve our readiness for future pandemics ${ }^{14}$.

\section{Online content}

Any methods, additional references, Nature Research reporting summaries, source data, extended data, supplementary information, acknowledgements, peer review information; details of author contributions and competing interests; and statements of data and code availability are available at https://doi.org/10.1038/s41586-020-2622-0.

1. Pallesen, J. et al. Immunogenicity and structures of a rationally designed prefusion MERS-CoV spike antigen. Proc. Natl Acad. Sci. USA 114, E7348-E7357 (2017).

2. Korber, B. et al. Tracking changes in SARS-CoV-2 spike: evidence that D614G increases infectivity of the COVID-19 virus. Cell 182, 812-827.e19 (2020).

3. Dong, E., Du, H. \& Gardner, L. An interactive web-based dashboard to track COVID-19 in real time. Lancet Infect. Dis. 20, 533-534 (2020).

4. Keni, R., Alexander, A., Nayak, P. G., Mudgal, J. \& Nandakumar, K. COVID-19: emergence, spread, possible treatments, and global burden. Front. Public Health 8, 216 (2020).

5. Graham, B. S. Rapid COVID-19 vaccine development. Science 368, 945-946 (2020).

6. Graham, B. S., Gilman, M. S. A. \& McLellan, J. S. Structure-based vaccine antigen design. Annu. Rev. Med. 70, 91-104 (2019).

7. McLellan, J. S. et al. Structure of RSV fusion glycoprotein trimer bound to a prefusion-specific neutralizing antibody. Science 340, 1113-1117 (2013).

8. McLellan, J. S. et al. Structure-based design of a fusion glycoprotein vaccine for respiratory syncytial virus. Science 342, 592-598 (2013).

9. Crank, M. C. et al. A proof of concept for structure-based vaccine design targeting RSV in humans. Science 365, 505-509 (2019).

10. Gilman, M. S. A. et al. Rapid profiling of RSV antibody repertoires from the memory B cells of naturally infected adult donors. Sci. Immunol. 1, eaaj1879 (2016).

11. Walls, A. C. et al. Cryo-electron microscopy structure of a coronavirus spike glycoprotein trimer. Nature 531, 114-117 (2016).

12. Kirchdoerfer, R. N. et al. Pre-fusion structure of a human coronavirus spike protein. Nature 531, 118-121 (2016).

13. Graham, B. S. \& Sullivan, N. J. Emerging viral diseases from a vaccinology perspective: preparing for the next pandemic. Nat. Immunol. 19, 20-28 (2018).
14. Graham, B. S. \& Corbett, K. S. Prototype pathogen approach for pandemic preparedness: world on fire. J. Clin. Invest. 130, 3348-3349 (2020).

15. Menachery, V. D. et al. A SARS-like cluster of circulating bat coronaviruses shows potential for human emergence. Nat. Med. 21, 1508-1513 (2015).

16. Menachery, V. D. et al. SARS-like WIV1-CoV poised for human emergence. Proc. Natl Acad. Sci. USA 113, 3048-3053 (2016)

17. Graham, B. S., Mascola, J. R. \& Fauci, A. S. Novel vaccine technologies: essential components of an adequate response to emerging viral diseases. J. Am. Med. Assoc. 319, 1431-1432 (2018).

18. Dowd, K. A. et al. Rapid development of a DNA vaccine for Zika virus. Science 354, 237-240 (2016).

19. Pardi, N., Hogan, M. J., Porter, F. W. \& Weissman, D. mRNA vaccines-a new era in vaccinology. Nat. Rev. Drug Discov. 17, 261-279 (2018).

20. Hassett, K. J. et al. Optimization of lipid nanoparticles for intramuscular administration of mRNA vaccines. Mol. Ther. Nucleic Acids 15, 1-11 (2019).

21. Mauger, D. M. et al. mRNA structure regulates protein expression through changes in functional half-life. Proc. Natl Acad. Sci. USA 116, 24075-24083 (2019).

22. Cockrell, A. S. et al. A mouse model for MERS coronavirus-induced acute respiratory distress syndrome. Nat. Microbiol. 2, 16226 (2016).

23. Wrapp, D. et al. Cryo-EM structure of the 2019-nCoV spike in the prefusion conformation. Science 367, 1260-1263 (2020).

24. Freeman, B. et al. Validation of a SARS-CoV-2 spike protein ELISA for use in contact investigations and serosurveillance. Preprint at https://doi.org/10.1101/2020.04.24.057323 (2020).

25. Klumpp-Thomas, C. et al. Standardization of enzyme-linked immunosorbent assays for serosurveys of the SARS-CoV-2 pandemic using clinical and at-home blood sampling. Preprint at https://doi.org/10.1101/2020.05.21.20109280 (2020).

26. Kim, H. W. et al. Respiratory syncytial virus disease in infants despite prior administration of antigenic inactivated vaccine. Am. J. Epidemiol. 89, 422-434 (1969).

27. Fulginiti, V. A., Eller, J. J., Downie, A. W. \& Kempe, C. H. Altered reactivity to measles virus. Atypical measles in children previously immunized with inactivated measles virus vaccines. J. Am. Med. Assoc. 202, 1075-1080 (1967).

28. Bolles, M. et al. A double-inactivated severe acute respiratory syndrome coronavirus vaccine provides incomplete protection in mice and induces increased eosinophilic proinflammatory pulmonary response upon challenge. J. Virol. 85, 12201-12215 (2011).

29. Czub, M., Weingartl, H., Czub, S., He, R. \& Cao, J. Evaluation of modified vaccinia virus Ankara based recombinant SARS vaccine in ferrets. Vaccine 23, 2273-2279 (2005).

30. Deming, D. et al. Vaccine efficacy in senescent mice challenged with recombinant SARS-CoV bearing epidemic and zoonotic spike variants. PLoS Med 3, E525 (2006)

31. Hou, Y.J. et al. SARS-CoV-2 reverse genetics reveals a variable infection gradient in the respiratory tract. Cell 182, 429-446 (2020).

32. Dinnon, K. H. et al. A mouse-adapted model of SARS-CoV-2 to test COVID-19 countermeasures. Nature https://doi.org/10.1038/s41586-020-2708-8 (2020).

33. Jackson, L. A. et al. An mRNA vaccine against SARS-CoV-2-preliminary report. N. Engl. J. Med. Moa2O22483 (2020)

Publisher's note Springer Nature remains neutral with regard to jurisdictional claims in published maps and institutional affiliations.

(c) This is a U.S. government work and not under copyright protection in the U.S.; foreign copyright protection may apply 2020 


\section{Methods}

\section{Data reporting}

No statistical methods were used to predetermine sample size. The experiments were not randomized. The investigators were not blinded to allocation during experiments and outcome assessment.

\section{Pre-clinical mRNA-1273 mRNA and LNP production process}

A sequence-optimized mRNA encoding SARS-CoV-2S(2P) protein was synthesized in vitro using an optimized T7 RNA polymerase-mediated transcription reaction with complete replacement of uridine by N1-methyl-pseudouridine ${ }^{34}$. The reaction included a DNA template containing the immunogen open reading frame flanked by $5^{\prime}$ untranslated region (UTR) and 3' UTR sequences and was terminated by an encoded polyA tail. After transcription, the Cap 1 structure was added to the $5^{\prime}$ end using vaccinia capping enzyme (New England Biolabs) and Vaccinia 2' $O$-methyltransferase (New England Biolabs). The mRNA was purified by oligo-dT affinity purification, buffer exchanged by tangential flow filtration into sodium acetate, $\mathrm{pH} 5.0$, sterile filtered, and kept frozen at $-20^{\circ} \mathrm{C}$ until further use.

The mRNA was encapsulated in a lipid nanoparticle through a modified ethanol-drop nanoprecipitation process as described previously ${ }^{20}$. In brief, ionizable, structural, helper and polyethylene glycol lipids were mixed with mRNA in acetate buffer, $\mathrm{pH}$ 5.0, at a ratio of 2.5:1 (lipids:mRNA). The mixture was neutralized with Tris- $\mathrm{Cl} \mathrm{pH} \mathrm{7.5,} \mathrm{sucrose}$ was added as a cryoprotectant, and the final solution was sterile filtered. Vials were filled with formulated LNP and stored frozen at $-70^{\circ} \mathrm{C}$ until further use. The drug product underwent analytical characterization, which included the determination of particle size and polydispersity, encapsulation, mRNA purity, double stranded RNA content, osmolality, $\mathrm{pH}$, endotoxin and bioburden, and the material was deemed acceptable for in vivo study.

\section{MERS-CoV and SARS-CoV protein expression and purification} Vectors encoding MERS-CoV S- $2 \mathrm{P}^{1}$ and SARS-CoV S- $2 \mathrm{P}^{23}$ were generated as previously described with the following small amendments. Proteins were expressed by transfection of plasmids into Expi293 cells using Expifectamine transfection reagent (ThermoFisher) in suspension at $37^{\circ} \mathrm{C}$ for $4-5$ days. Transfected cell culture supernatants were collected, buffer exchanged into $1 \times$ PBS, and protein was purified using Strep-Tactin resin (IBA). For proteins used for mouse inoculations, tags were cleaved with addition of HRV3C protease (ThermoFisher) (1\%wt/wt) overnight at $4{ }^{\circ} \mathrm{C}$. Size-exclusion chromatography using Superose 6 Increase column (GE Healthcare) yielded final purified protein.

\section{Design and production of recombinant minifibritin foldon protein}

A mammalian codon-optimized plasmid encoding foldon inserted minifibritin (ADIVLNDLPFVDGPPAEGQSRISWIKNGEEILGADTQYGSE GSMNRPTVSVLRNVEVLDKNIGILKTSLETANSDIKTIQEAGYIPEAPRDGQA YVRKDGEWVLLSTFLSPALVPRGSHHHHHHSAWSHPQFEK) with a C-terminal thrombin cleavage site, $6 \times$ His tag, and Strep-TagIl was synthesized and subcloned into a mammalian expression vector derived from pLEXm. The construct was expressed by transient transfection of Expi293 (ThermoFisher) cells in suspension at $37^{\circ} \mathrm{C}$ for 5 days. The protein was first purified with a $\mathrm{Ni}^{2+}$-nitrilotriacetic acid resin (GE Healthcare) using an elution buffer consisting of $50 \mathrm{mM}$ Tris- $\mathrm{HCl}, \mathrm{pH} 7.5,400$ $\mathrm{mM} \mathrm{NaCl}$ and $300 \mathrm{mM}$ imidazole $\mathrm{pH} 8.0$, followed by purification with StrepTactin resin (IBA) according to the manufacturer's instructions.

\section{Cell lines}

HEK293T/17 (ATCC CRL-11268), Vero E6 (ATCC), Huh7.5 cells (provided by D. R. Taylor, US Food and Drug Administration) and ACE2-expressing 293T cells (provided by M. Farzan, Scripps Research Institute) were cultured in Dulbecco's modified Eagle's medium (DMEM) supplemented with $10 \% \mathrm{FBS}, 2 \mathrm{mM}$ glutamine and $1 \%$ penicillin-streptomycin at $37^{\circ} \mathrm{C}$ and $5 \% \mathrm{CO}_{2}$. Vero $\mathrm{E} 6$ cells used in plaque assays to determine lung and nasal turbinate viral titres were cultured in DMEM supplemented with $10 \%$ Fetal Clone II and $1 \%$ antibiotic-antimycotic at $37^{\circ} \mathrm{C}$ and $5 \% \mathrm{CO} 2$. Vero E6 cells used in plaque-reduction neutralization test (PRNT) assays were cultured in DMEM supplemented with $10 \%$ Fetal Clone II and amphotericin $\mathrm{B}\left(0.25 \mu \mathrm{g} \mathrm{ml}^{-1}\right)$ at $37^{\circ} \mathrm{C}$ and $5 \% \mathrm{CO}_{2}$. Lentivirus encoding hACE2-P2A-TMPRSS2 was made to generate A549-hACE2-TMPRSS2 cells, which were maintained in DMEM supplemented with $10 \%$ FBS and $1 \mu \mathrm{g} \mathrm{ml}^{-1}$ puromycin. Expi293 cells were maintained in the manufacturer's suggested medium. BHK-21/WI-2 cells were obtained from Kerafast and cultured in DMEM with $5 \% \mathrm{FBS}$ at $37^{\circ} \mathrm{C}$ and $6-8 \% \mathrm{CO}_{2}$. Cell lines were not authenticated. All cells lines were tested for mycoplasma and remained negative.

\section{In vitro mRNA expression}

HEK293T cells were transiently transfected with mRNA encoding SARS-CoV-2 wild-type S or S(2P) protein using a TranIT mRNA transfection kit (Mirus). After $24 \mathrm{~h}$, the cells were collected and resuspended in fluorescence-activated cell sorting (FACS) buffer (1× PBS, 3\% FBS, $0.05 \%$ sodium azide). To detect surface-protein expression, the cells were stained with $10 \mu \mathrm{g} \mathrm{ml}^{-1} \mathrm{ACE} 2-$ Flag (Sigma) or $10 \mu \mathrm{g} \mathrm{ml}^{-1} \mathrm{CR} 3022^{35}$ in FACS buffer for $30 \mathrm{~min}$ on ice. Thereafter, cells were washed twice in FACS buffer and incubated with FITC-anti-Flag (Sigma) or Alexa Fluor 647-goat anti-human IgG (Southern Biotech) in FACS buffer for $30 \mathrm{~min}$ on ice. Live/Dead aqua fixable stain (Invitrogen) were used to assess viability. Data acquisition was performed on a BD LSRII Fortessa instrument (BD Biosciences) and analysed by FlowJo software v.10 (Tree Star).

\section{Mouse models}

Animal experiments were carried out in compliance with all pertinent US National Institutes of Health regulations and approval from the Animal Care and Use Committee (ACUC) of the Vaccine Research Center, Moderna Inc., or University of North Carolina at Chapel Hill. For immunogenicity studies, 6- to 8-week-old female BALB/c (Charles River), BALB/cJ, C57BL/6J or B6C3F1/J mice (Jackson Laboratory) were used. mRNA formulations were diluted in $50 \mu \mathrm{l} 1 \times \mathrm{PBS}$, and mice were inoculated intramuscularly in the same hind leg for both prime and boost. Control mice received PBS because previous studies have demonstrated the mRNA formulations being tested do not create substantial levels of nonspecific immunity beyond a few days ${ }^{36-38}$. For all SARS-CoV-2 S(2P) protein vaccinations, mice were inoculated intramuscularly with SAS as previously described ${ }^{1}$. For $\mathrm{S}(2 \mathrm{P})+$ alum immunizations, SARS-CoV-2S $(2 \mathrm{P})$ protein $+250 \mu$ g alum hydrogel was delivered intramuscularly. For challenge studies to evaluate MERS-CoV vaccines, 16 - to 20 -week-old male and female $288 / 330^{+/+}$mice $^{22}$ were immunized. Four weeks post-boost, pre-challenge sera were collected from a subset of mice, and the remaining mice were challenged with $5 \times 10^{5}$ PFU of a mouse-adapted MERS-CoV EMC derivative, $\mathrm{m} 35 \mathrm{c}^{39}$. On day 3 post-challenge, lungs were collected and haemorrhage and viral titre were assessed according to previously published methods ${ }^{40}$. For challenge studies to evaluate SARS-CoV-2 vaccines, BALB/cJ mice were challenged with $10^{5}$ PFU SARS-CoV-2 MA. This virus contains two mutations (Q498T/P499Y) in the receptor binding domain that enable binding of SARS-CoV-2S protein to the mouse ACE2 receptor and infection and replication in the upper and lower respiratory tract ${ }^{32}$. On day 2 post-challenge, lungs and nasal turbinates were collected for viral titre assessment according to previously published methods ${ }^{32}$. Sample size for animal experiments was determined on the basis of criteria set by institutional ACUC. Experiments were not randomized or blinded.

\section{Histology}

Lungs were collected from mice at the indicated study end points and placed in $10 \%$ neutral-buffered formalin until adequately fixed. Thereafter, tissues were trimmed to a thickness of $3-5 \mathrm{~mm}$, processed and paraffin 
embedded. The respective paraffin tissue blocks were sectioned at $5 \mu \mathrm{m}$ and stained with haematoxylin and eosin. All sections were examined by a board-certified veterinary pathologist using an Olympus BX51 light microscope, and photomicrographs were taken using an Olympus DP73 camera.

\section{ELISA}

Nunc Maxisorp ELISA plates (ThermoFisher) were coated with $100 \mathrm{ng}$ per well of protein in $1 \times \mathrm{PBS}$ at $4{ }^{\circ} \mathrm{C}$ for $16 \mathrm{~h}$. Where applicable, to eliminate fold-on-specific binding from MERS-CoVS(2P) or SARS-CoV-2S(2P) protein-immune mouse serum, $50 \mathrm{\mu g} \mathrm{ml}^{-1}$ of fold-on protein was added for $1 \mathrm{~h}$ at room temperature. After standard washes and blocks, plates were incubated with serial dilutions of heat-inactivated sera for $1 \mathrm{~h}$ at room temperature. Following washes, anti-mouse IgG, IgG1 or IgG2a and/or IgG2c-horseradish peroxidase conjugates (ThermoFisher) were used as secondary antibodies, and 3,5,3'5'-tetramethylbenzidine (TMB) (KPL) was used as the substrate to detect antibody responses. End-point titres were calculated as the dilution that emitted an optical density exceeding $4 \times$ background (secondary antibody alone).

\section{Lentivirus-based pseudovirus-neutralization assay}

The pseudovirus-neutralization assay measures the inhibition of pseudovirus attachment and entry including fusion-inhibiting activity. It is a single-round virus, does not replicate, and does not express the S protein in transduced cells. Therefore, pseudovirus infection will not cause cell-to-cell fusion or plaque formation that can be measured in a classical neutralization assay using live virus. This pseudovirus neutralization assay has been shown to correlate with live virus plaque-reduction neutralization ${ }^{33}$, and because it does not require BL3 containment, was chosen as the preferred assay for measuring neutralizing activity in these studies. We introduced divergent amino acids, as predicted from translated sequences, into the CMV/R-MERS-CoV EMC S (GenBank: AFS88936) gene ${ }^{41}$ to generate a MERS-CoV m $35 \mathrm{c} 4$ S gene $^{39}$. To produce SARS-CoV-2 pseudoviruses, a codon-optimized CMV/R-SARS-CoV-2 S (Wuhan-1, GenBank: MN908947.3) plasmid was constructed. Pseudoviruses were produced by co-transfection of plasmids encoding a luciferase reporter, lentivirus backbone, and S genes into HEK293T/17 cells (ATCC CRL-11268), as previously described ${ }^{41}$. For SARS-CoV-2 pseudovirus, human transmembrane protease serine 2 (TMPRSS2) plasmid was also co-transfected ${ }^{42}$. Pseudoneutralization assay methods have been previously described ${ }^{1,33}$. In brief, heat-inactivated serum was mixed with pseudoviruses, incubated, and then added to Huh7.5 cells or ACE-2-expressing 293T cells for MERS-CoV and SARS-CoV-2 respectively. Seventy-two hours later, cells were lysed and luciferase activity (in relative light units (RLU)) was measured. Per cent neutralization was normalized considering uninfected cells as $100 \%$ neutralization and cells infected with only pseudovirus as $0 \%$ neutralization. $\mathrm{IC}_{50}$ titres were determined using a log (agonist) vs normalized-response (variable slope) nonlinear function in Prism v8 (GraphPad).

\section{Recombinant VSV $\Delta$ G-based pseudovirus neutralization assay}

Codon-optimized wild-type (D614) or D614G spike gene (Wuhan-Hu-1 strain, NCBI reference sequence:NC_045512.2) was cloned into pCAGGS vector. Togenerate VSV $\Delta$ G-based SARS-CoV-2 pseudovirus, BHK-21/WI-2 cells were transfected with the spike expression plasmid and infected VSV $\Delta G$-firefly-luciferase as previously described ${ }^{43}$. A549-hACE2-TMPRSS2 cells were infected by pseudovirus for $1 \mathrm{~h}$ at $37^{\circ} \mathrm{C}$. The inoculum virus or virus-antibody mix was removed after infection. Eighteen hours later, an equal volume of One-Glo reagent (Promega) was added to culture medium for readout using a BMG PHERastar-FS plate reader. The neutralization procedure and data analysis are the same as mentioned above for the lentivirus-based pseudovirus neutralization assay.

\section{PRNT assays}

Heat-inactivated sera were diluted in gelatin saline $(0.3 \%$ (wt/vol) gelatin in PBS supplemented with $\mathrm{CaCl}_{2}$ and $\mathrm{MgCl}_{2}$ to generate a 1:5 dilution of the original specimen, which served as a starting concentration for further serial $\log _{4}$ dilutions terminating in $1: 81,920$. Sera were combined with an equal volume of SARS-CoV-2 clinical isolate 2019-nCoV USA-WA1-F6/2020 in gelatin saline, resulting in an average concentration of 730 PFU per $\mathrm{ml}$ (determined from plaque counts of 24 individual wells of untreated virus) in each serum dilution. Thus, final serum concentrations ranged from 1:10 to 1:163,840 of the original. Virus-serum mixtures were incubated for $20 \mathrm{~min}$ at $37^{\circ} \mathrm{C}$, followed by adsorption of $0.1 \mathrm{ml}$ to each of two confluent Vero E6 cell monolayers (in $10 \mathrm{~cm}^{2}$ wells) for $30 \mathrm{~min}$ at $37^{\circ} \mathrm{C}$. Cell monolayers were overlaid with DMEM containing $1 \%$ agar and incubated for $3 \mathrm{~d}$ at $37^{\circ} \mathrm{C}$ in humidified $5 \% \mathrm{CO}_{2}$. Plaques were enumerated by direct visualization. The average number of plaques in virus + serum (duplicate) and virus-only (24 repeats) wells was used to generate percent neutralization curves according the following formula: 1 - (ratio of mean number of plaques in the presence and absence of serum). The PRNT IC ${ }_{50}$ titre was defined as the reciprocal serum dilution at which the neutralization curve crossed the 50\% threshold.

\section{Intracellular cytokine staining}

Mononuclear single-cell suspensions from whole mouse spleens were generated using a gentleMACS tissue dissociator (Miltenyi Biotec) followed by $70-\mu \mathrm{m}$ filtration and density gradient centrifugation using Fico/Lite-LM medium (Atlanta Biologicals). Cells from each mouse were resuspended in R10 media (RPMI 1640 supplemented with penicillin-streptomycin antibiotic, $10 \%$ heat-inactivated FBS, Glutamax and HEPES) and incubated for $6 \mathrm{~h}$ at $37^{\circ} \mathrm{C}$ with protein transport inhibitor cocktail (eBioscience) under three conditions: no peptide stimulation, and stimulation with two S-protein peptide pools (JPT product PM-WCPV-S-1). Peptide pools were used at a final concentration of 2 $\mu \mathrm{g} \mathrm{ml}$ per peptide. Cells from each group were pooled for stimulation with cell stimulation cocktail (eBioscience) as a positive control. Following stimulation, cells were washed with PBS before staining with LIVE/DEAD Fixable Blue Dead Cell Stain (Invitrogen, L23105; 1:800) for 20 min at room temperature. Cells were then washed in FC buffer (PBS supplemented with 2\% heat-inactivated FBS and $0.05 \% \mathrm{NaN}_{3}$ ) and resuspended in Fc Block (BD, 553141, clone 2.4G2; 1:100) for 5 min at room temperature before staining with a surface stain cocktail containing the following antibodies: I-A/I-E PE (BD, 557000, clone M5/114.15.2; 1:2,500), CD8a BUV805 (BD, 612898, clone 53-6.7;1:80), CD44 BUV395 (BD, 740215, clone IM7;1:800), CD62L BV605 (Biolegend, 104418, clone MEL-14; 1:5,000) and CD4 BV480 (BD, 565634, clone RM4-5; 1:500) in brilliant stain buffer (BD). After 15 min, cells were washed with FC buffer then fixed and permeabilized using the BD Cytofix/Cytoperm fixation/permeabilization solution kit according to the manufacturer's instructions. Cells were washed in perm/wash solution and stained with Fc Block ( 5 min at room temperature), followed by intracellular staining $\left(30\right.$ min at $\left.4{ }^{\circ} \mathrm{C}\right)$ using a cocktail of the following antibodies: CD3e BUV737 (BD, 741788, clone 17A2; 1:80), IFN- $\gamma$ BV650 (BD, 563854, clone XMG1.2; 1:500), TNF BV711 (BD, 563944, clone MP6-XT22; 1:80), IL-2 BV421 (BD, 562969, cloneJES6-5H4;1:80), IL-4 Alexa Fluor 488 (Biolegend, 504109, clone 11B11;1:80) and IL-5 APC (Biolegend, 504306, clone TRFK5;1:320) in $1 \times$ perm/wash diluted with brilliant stain buffer. Finally, cells were washed in perm/wash solution and resuspended in $0.5 \%$ PFA-FC stain buffer before running on a Symphony A5 flow cytometer (BD). Analysis was performed using FlowJo software, v.10.6.2 according to the gating strategy outlined in Extended Data Fig. 10. Background cytokine expression in the no-peptide condition was subtracted from that measured in the S1 and S2 peptide pools for each individual mouse.

\section{T cell stimulation and cytokine analysis}

Spleens from immunized mice were collected two weeks post-boost. Two-million splenocytes per well (96-well plate) were stimulated in vitro with two peptide libraries, JPT1 and JPT2, (15mers with 11 amino acid overlap) covering the entire SARS-CoV-2 S protein (JPT product 
PM-WCPV-S-1). Both peptide libraries were used at a final concentration of $1 \mu \mathrm{g} \mathrm{ml}{ }^{-1}$. After $24 \mathrm{~h}$ of culture at $37^{\circ} \mathrm{C}$, the plates were centrifuged and supernatant was collected and frozen at $-80^{\circ} \mathrm{C}$ for cytokine detection. Measurements and analyses of secreted cytokines from a murine 35-plex kit were performed using a multiplex bead-based technology (Luminex) assay with a Bio-Plex 200 instrument (Bio-Rad) after twofold dilution of supernatants.

\section{Statistical analysis}

Geometric means or arithmetic means are represented by the heights of bars, or symbols, and error bars represent the corresponding s.d. Dotted lines indicate assay limits of detection. Two-sided Mann-Whitney $U$-tests were used to compare two experimental groups and two-sided Wilcoxon signed-rank tests to compare the same animals at different time points. To compare more than two experimental groups, KruskalWallis ANOVA with Dunn's multiple comparisons tests were applied. In Extended Data Fig. 5a, b, all doses were compared to the $20 \mu \mathrm{g}$ dose by two-sided Mann-Whitney $U$-test in a stepwise fashion, such that lowest doses were tested first at $\alpha=0.05$ and higher doses were tested only if the lower doses were significant. In Extended Data Fig. 5c, a Spearman correlation test was used to correlate binding antibody titres to pseudovirus-neutralizing antibody titres. Statistical analyses were performed using R v.4.0.0 or Prism v.8 (GraphPad). ${ }^{*} P<0.05,{ }^{* *} P<0.01$, ${ }^{* * *} P<0.001,{ }^{* * * *} P<0.0001$.

\section{Reporting summary}

Further information on research design is available in the Nature Research Reporting Summary linked to this paper.

\section{Data availability}

The authors declare that the data supporting the findings of this study are available within this Article and its Supplementary Information. Source data are provided with this paper.

34. Nelson, J. et al. Impact of mRNA chemistry and manufacturing process on innate immune activation. Sci. Adv. 6, eaaz6893 (2020)

35. ter Meulen, J. et al. Human monoclonal antibody combination against SARS coronavirus: synergy and coverage of escape mutants. PLoS Med. 3, e237 (2006).

36. John, S. et al. Multi-antigenic human cytomegalovirus mRNA vaccines that elicit potent humoral and cell-mediated immunity. Vaccine 36, 1689-1699 (2018).

37. Bahl, K. et al. Preclinical and clinical demonstration of immunogenicity by mRNA vaccines against $\mathrm{H} 10 \mathrm{~N} 8$ and $\mathrm{H} 7 \mathrm{~N} 9$ influenza viruses. Mol. Ther. 25, 1316-1327 (2017)

38. Vogel, A. B. et al. Self-amplifying RNA vaccines give equivalent protection against influenza to mRNA vaccines but at much lower doses. Mol. Ther. 26, 446-455 (2018).
39. Douglas, M. G., Kocher, J. F., Scobey, T., Baric, R. S. \& Cockrell, A. S. Adaptive evolution influences the infectious dose of MERS-CoV necessary to achieve severe respiratory disease. Virology 517, 98-107 (2018).

40. Scobey, T. et al. Reverse genetics with a full-length infectious cDNA of the Middle East respiratory syndrome coronavirus. Proc. Natl Acad. Sci. USA 110, 16157-16162 (2013)

41. Wang, L. et al. Evaluation of candidate vaccine approaches for MERS-CoV. Nat. Commun. 6, 7712 (2015).

42. Böttcher, E. et al. Proteolytic activation of influenza viruses by serine proteases TMPRSS2 and HAT from human airway epithelium. J. Virol. 80, 9896-9898 (2006).

43. Whitt, M. A. Generation of VSV pseudotypes using recombinant $\Delta G$-VSV for studies on virus entry, identification of entry inhibitors, and immune responses to vaccines. J. Virol. Methods 169, 365-374 (2010).

Acknowledgements We thank K. Bok, K. Carlton, M. Kanekiyo, R. Seder and other members of all included laboratories for critical discussions, advice and review of the manuscript; J. Stein for technology transfer; M. Young for administrative support; members of the NIH NIAID VRC Translational Research Program for technical assistance with mouse experiments; B. Hartman for assistance with graphics; H. Mu and M. Farzan for the ACE2-overexpressing 293 cells; and M. Whitt for support on VSV-based pseudovirus production. This work was supported by the Intramural Research Program of the VRC and the Division of Intramural Research, NIAID, NIH (B.S.G.), NIH NIAID grant R01-AI127521 (J.S.M.), and NIH grants Al149644 and Al100625 (R.S.B.) mRNA-1273 has been funded in part with Federal funds from the Department of Health and Human Services, Office of the Assistant Secretary for Preparedness and Response, Biomedical Advanced Research and Development Authority, under contract 75A50120C00034. PRNT assays were funded under NIH contract HHSN261200800001E agreement 17x198 (to J.D.C.), furnished through Leidos Biomedical Research. MERS-CoV mRNA mouse challenge studies were funded under $\mathrm{NIH}$ contract HHSN272201700036I task order no. 75N93019F00132 requisition no. 5494549 (to R.S.B.). K.S.C.'s research fellowship was partially funded by the Undergraduate Scholarship Program, Office of Intramural Training and Education, Office of the Director, NIH. D.R.M. was funded by NIH NIAID grant T32-AI007151 and a Burroughs Wellcome Fund Postdoctoral Enrichment Program Award.

Author contributions K.S.C., D.K.E., S.R.L., O.M.A, S.B.-B., R.A.G., S.H., A.S., C.T.Z, A.T.D., K.H.D. S.M.E., C.A.S., A. Woods, E.J.F., D.R.M., K.W.B., M. Minai, B.M.N., G.B.H., K.W., C.H., K.B., D.G.-D., L.M., I.R., W.-P.K., S.D.S., L.W., Y.Z., E.P., L.A.C., R.J.L., N.E.A., E.N., M. Metkar, V.P., C.L., M.K.L., W.S., K.L., E.S.Y, A. West, K.L.G., L.J.S., N.A.D.-R., G.S.-J., H.B., G.S.A., M.C.N., T.J.R., M.R.D., J.D.C. I.N.M., K.M.M., J.R.M., R.S.B., A.C. and B.S.G. designed, completed and/or analysed experiments. K.S.C., O.M.A., G.B.H., N.W., D.W., J.S.M. and B.S.G. contributed new reagents and/ or analytic tools. K.S.C., K.M.M. and B.S.G. wrote the manuscript. All authors contributed to discussions in regard to and editing of the manuscript.

Competing interests K.S.C., N.W., J.S.M. and B.S.G. are inventors on International Patent Application no. WO/2018/081318 entitled 'Prefusion Coronavirus Spike Proteins and Their Use'. K.S.C., O.M.A., G.B.H., N.W., D.W., J.S.M. and B.S.G. are inventors on US Patent Application no. 62/972,886 entitled '2019-nCoV Vaccine'. R.S.B. filed an invention report for the SARS-CoV-2 MA virus (UNC ref. 18752)

Additional information Supplementary information is available for this paper at https://doi.org/10.1038/s41586-0202622-0.

Correspondence and requests for materials should be addressed to A.C. or B.S.G. Peer review information Nature thanks Rino Rappuoli, Patrick C. Wilson and the other, anonymous, reviewer(s) for their contribution to the peer review of this work. Peer reviewer reports are available.

Reprints and permissions information is available at http://www.nature.com/reprints. 
a

MERS S-2P TM vs. Secreted MERS S-2P-foldon RNA

Neutralizing Antibody Responses

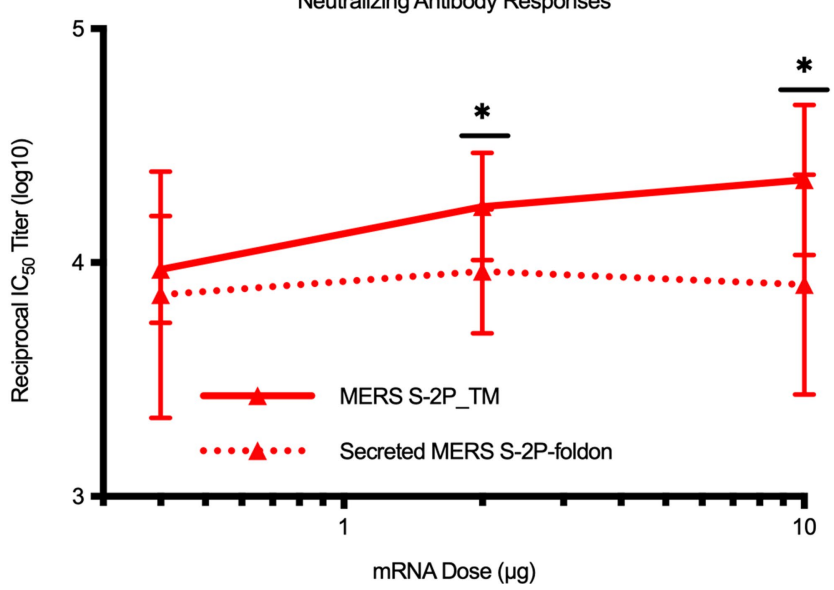

Extended Data Fig. 1 | Transmembrane-anchored MERS-CoV S-2P(S-2P_TM) mRNA elicits more potent pseudovirus neutralizing antibody responses than secreted MERS-CoV S(2P) and S WT mRNA. a, b, C57BL/6J mice $(n=10 /$ group) were immunized at weeks 0 and 4 with (a) $0.4,2$, or $10 \mu$ g of MERS-CoV S-2P_TM (red) or MERS S-2P_secreted (red hashed) or (b) $0.016 \mu \mathrm{g}, 0.08 \mu \mathrm{g}$, or b

MERS S-2P vs. MERS S WT mRA

Neutralizing Antibody Responses

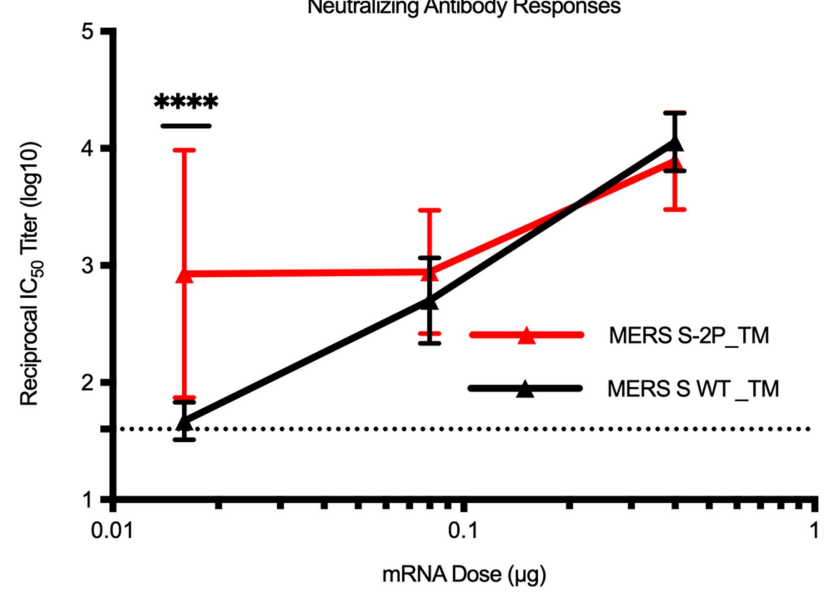

$0.4 \mu$ g of MERS-CoV S(2P) or MERS-CoVS WT_TM (black) ERNA. Ser were collected 4 weeks post-boost and assessed for neutralizing antibodies against MERS-CoV m35c4 pseudovirus. Immunogens were compared at each dose level by two-sided Mann-Whitney $U$-test. ${ }^{*} P<0.05,{ }^{* * * *} P<0.0001$. Data are presented as GMT \pm geometric sid. 


\section{Article}

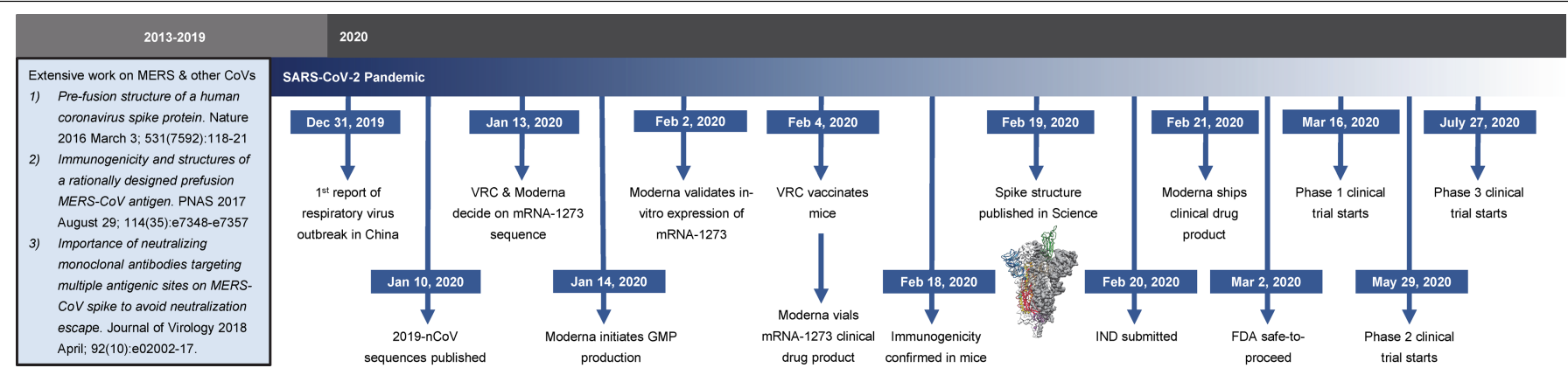

Extended Data Fig. 2 | Timeline for mRNA-1273's progression to clinical

trial. The morning after novel coronavirus ( $\mathrm{nCoV}$ ) sequences were released,

clinically-relevant mRNA-1273 was received to initiate animal experiments.

spike sequences were modified to include prefusion stabilizing mutations and Immunogenicity in mice was confirmed 15 days later. Moderna shipped clinical synthesized for protein production, assay development, and vaccine development. Twenty-five days after viral sequences were released, drug product 41 days after GMP production began, leading to the phase I clinical trial starting 66 days following the release of $\mathrm{nCoV}$ sequences. 
a

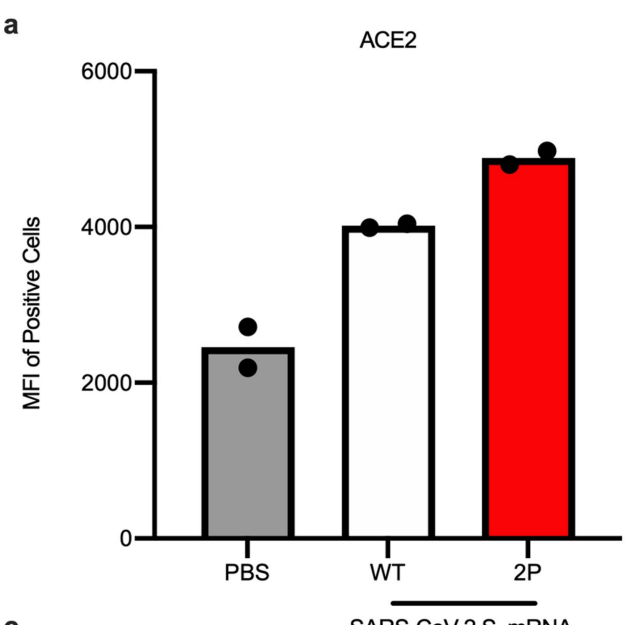

c

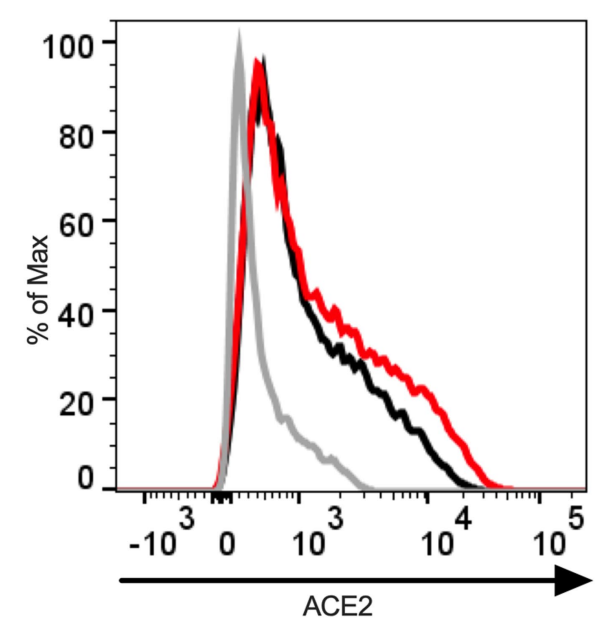

Extended Data Fig. 3 | In vitro expression of SARS-CoV-2 spike mRNA on the cell surface. $\mathbf{a}-\mathbf{d}$, 293T cells were transfected in duplicate with mRNA expressing SARS-CoV-2 wild-type spike (white bars, black lines) or S-2P (red),

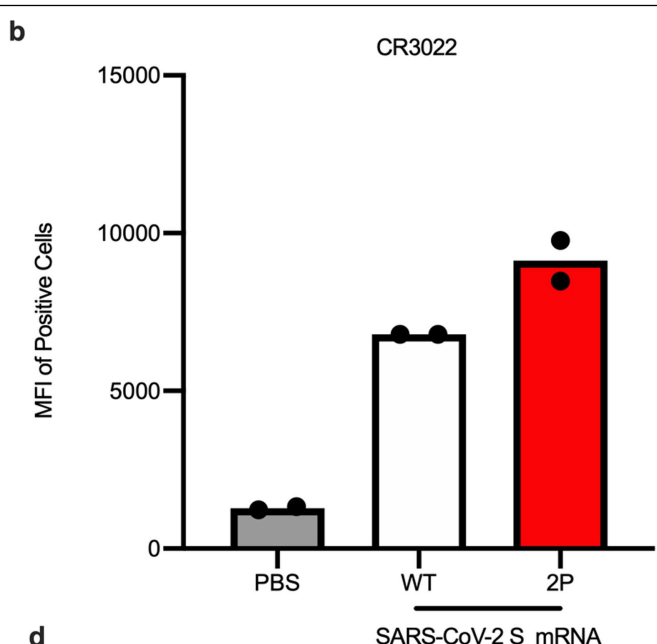

d

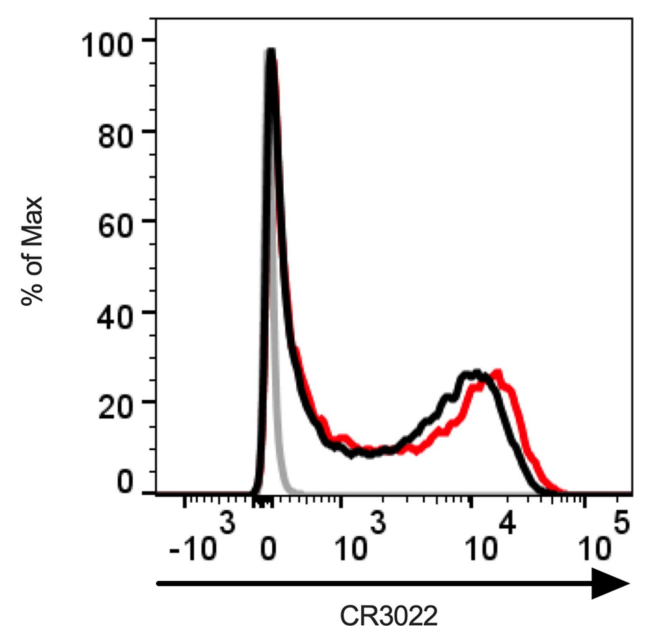

stained with ACE2 (a, c) or CR3022 (b, d), and evaluated by flow cytometry 24 post-transfection. Mock-transfected (PBS) cells served as a control (grey). $(\mathbf{a}, \mathbf{b})$ Data are presented as mean. 


\section{Article}

Neutralizing Antibodies

SARS-CoV-2 D614 vs D614G Strains

*

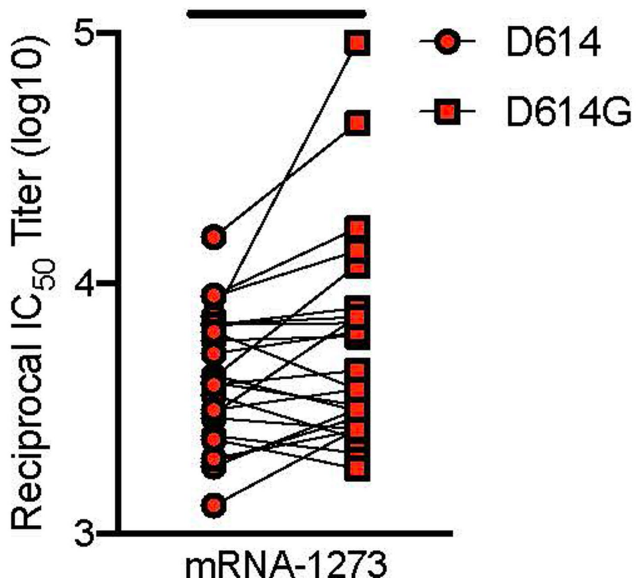

Extended Data Fig. 4 | mRNA-1273 elicits robust pseudovirus neutralizing antibody responses to SARS-CoV-2_D614G. BALB/c mice $(n=24)$ were

immunized at weeks 0 and 3 weeks with $1 \mu \mathrm{g}$ (red) of mRNA-1273, in three individual studies ( $n=8 /$ study). Sera were collected 2 weeks post-boost and assessed for neutralizing antibodies against homotypic SARS-CoV-2_D614 pseudovirus (circles) or SARS-CoV-2_D614G (squares). Comparisons between D614 and D614G were made by two-sided Wilcoxon signed rank test. ${ }^{*} P<0.05$. 


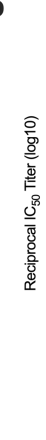
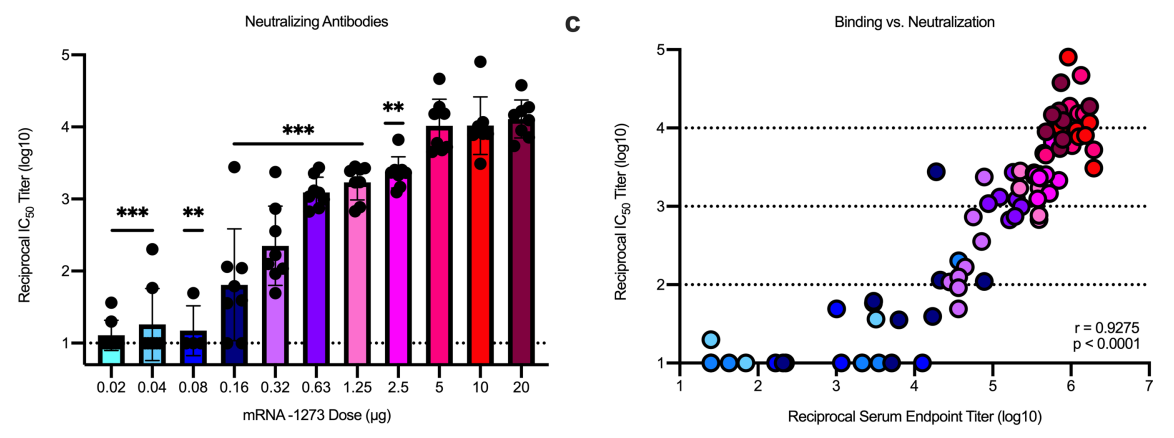

Extended Data Fig. 5 | Dose-dependent mRNA-1273-elicited antibody responses reveal strong positive correlation between binding and pseudovirus neutralization titres. a-c, BALB/c mice ( $n=10 /$ group) were immunized at weeks 0 and 3 weeks with various doses $(0.0025-20 \mu \mathrm{g})$ of mRNA-1273. Sera were collected 2 weeks post-boost and assessed for SARS-CoV-2S-specific IgG by ELISA (a) and neutralizing antibodies against homotypic SARS-CoV-2 pseudovirus (b).a, b, All doses were compared to the
$20 \mu$ g dose by two-sided Mann-Whitney $U$-test in a stepwise fashion, such that lowest doses were tested first at $\alpha=0.05$ and higher doses tested only if the lower doses were significant. Data are presented as GMT \pm geometric s.d., and dotted lines represent assay limits of detection. c, Spearman correlation test was used to correlate binding antibody titres to pseudovirus neutralizing antibody titres $(P<0.0001)$. Each dot represents an individual mouse. Dotted lines highlight $\log _{10} \mathrm{IC}_{50}$ boundaries. ${ }^{* *} P<0.01,{ }^{* * *} P<0.001$. 
Binding Antibodies

$* * * *$

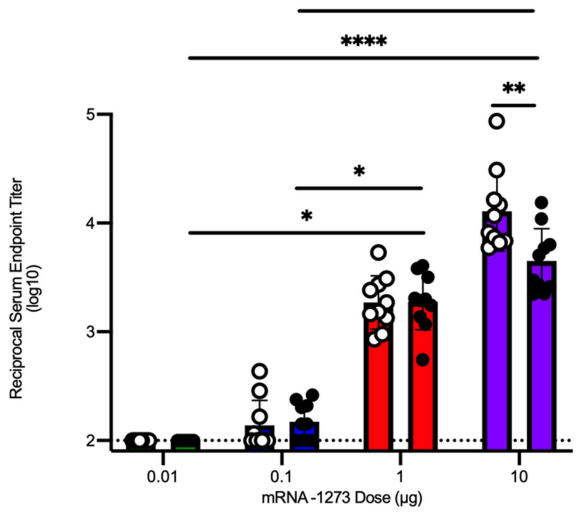

b

Neutralizing Antibodies

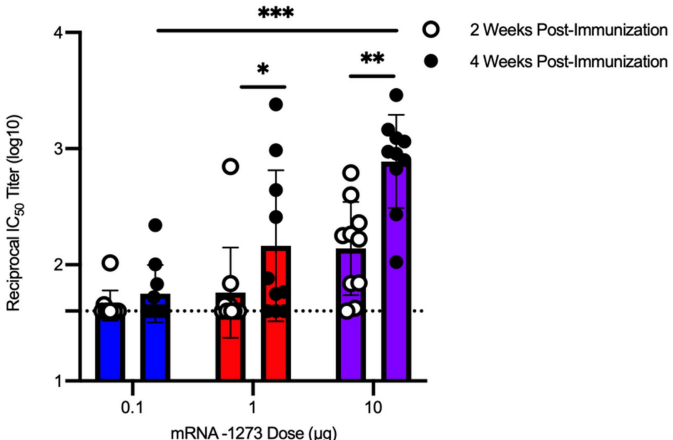

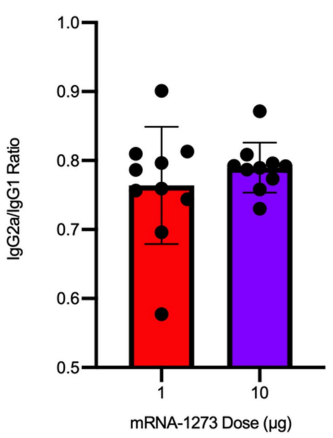

Extended Data Fig. 6 | A single dose of mRNA-1273 elicits robust antibody responses. a-c, BALB/cJ mice ( $n=10 /$ group) were immunized with 0.01 (green), 0.1 (blue), $1 \mu \mathrm{g}$ (red), or $10 \mu \mathrm{g}$ (purple) of mRNA-1273. Sera were collected 2 (unfilled circles) and 4 (filled circles) weeks post-immunization and assessed for SARS-CoV-2S-specific total IgG by ELISA (a) and neutralizing antibodies against homotypic SARS-CoV-2 pseudovirus (b).c, S-specific IgG2a and IgG1 were also measured by ELISA, and IgG2a to IgG1 subclass ratios were calculated. In a, b, Time points were compared within each dose level by two-sided Wilcoxon signed-rank test, and doses were compared 4 weeks post-boost by Kruskal-Wallis ANOVA with Dunn's multiple comparisons test. ${ }^{*} P<0.05,{ }^{* *} P<0.01,{ }^{* * *} P<0.001,{ }^{* * * *} P<0.0001$. c, Doses were compared by two-sided Mann-Whitney $U$-test, and no significance was found. Data are presented as GMT \pm geometric s.d. $(\mathbf{a}, \mathbf{b})$ or mean \pm s.d. (c), and dotted lines represent assay limits of detection. 


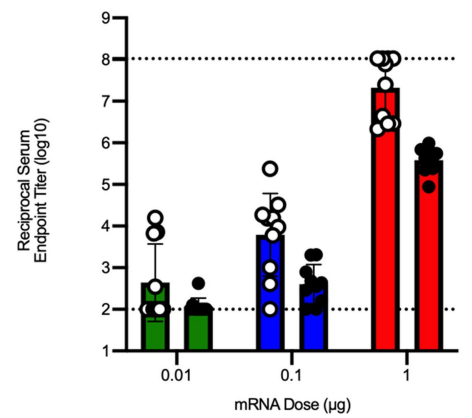

d

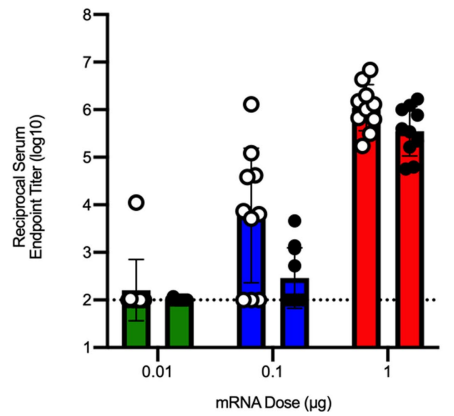

mRNA-1273
C57BL/6

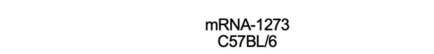

SARS-CoV-2 S-2P Protein + SAS
C

$\underset{\text { BALB/c }}{\lg \text { G2aatio }}$

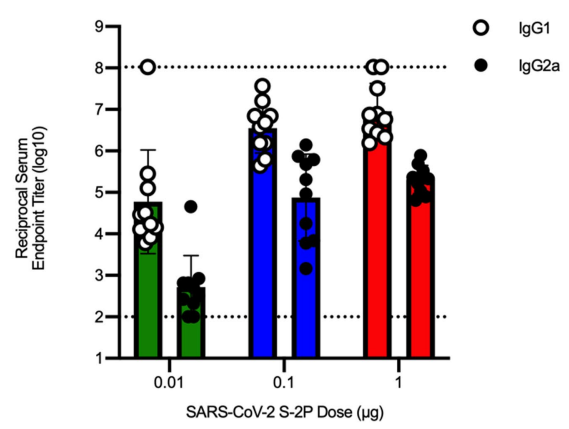

e

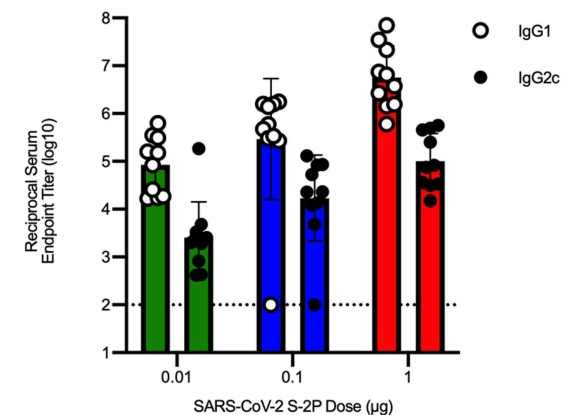

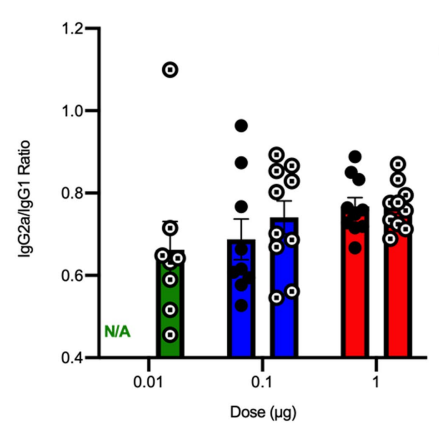

IgG2C/lgG1 Ratio
C57BL/6

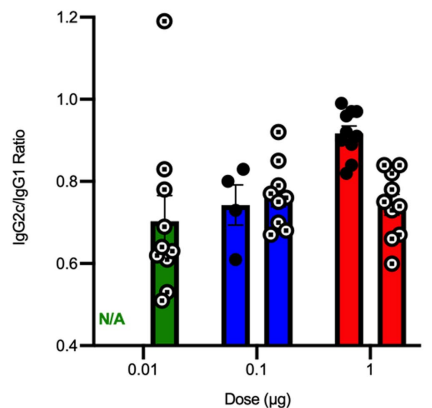

Extended Data Fig. 7 | mRNA-1273 and SAS-adjuvanted S-2P protein elicit both IgG2a and IgG1 subclass $S$-binding antibodies. $a-f, B A L B / c J(a-c)$ or C57BL/6J (d-f) mice ( $n=10$ /group) were immunized at weeks 0 and 3 with 0.01 (green), 0.1 (blue), or $1 \mu \mathrm{g}$ (red) of mRNA-1273 or SARS-CoV-2S-2P protein adjuvanted with SAS. Sera were collected 2 weeks post-boost and assessed by ELISA for SARS-CoV-2S-specific IgG1 and IgG2a or IgG2c for BALB/cJ and
C57BL/6J mice, respectively. End-point titres (a, b, d, e) and end-point titre ratios of IgG2a to IgG1 (c) and IgG2c to IgG1 (f) were calculated. For mice for which end-point titres did not reach the lower limit of detection (dotted line), ratios were not calculated (N/A). Data are presented as GMT \pm geometric s.d. $(\mathbf{a}, \mathbf{b}, \mathbf{d}, \mathbf{e})$ or mean \pm s.d. $(\mathbf{c}, \mathbf{f})$. 


\section{Article}

a

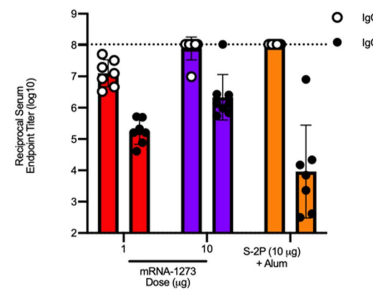

b

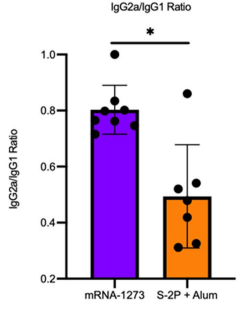

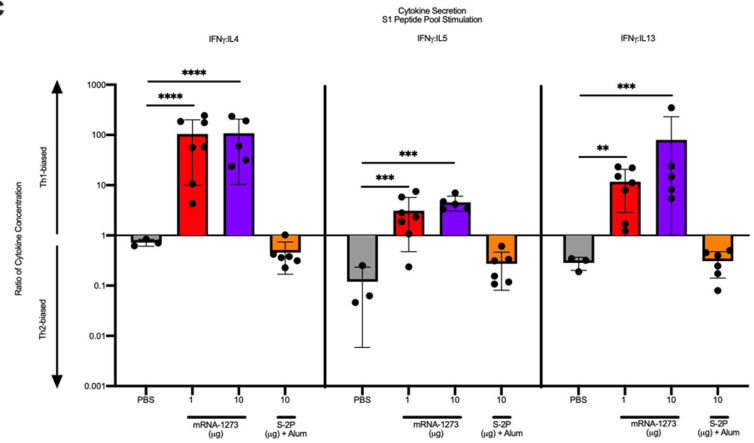

d
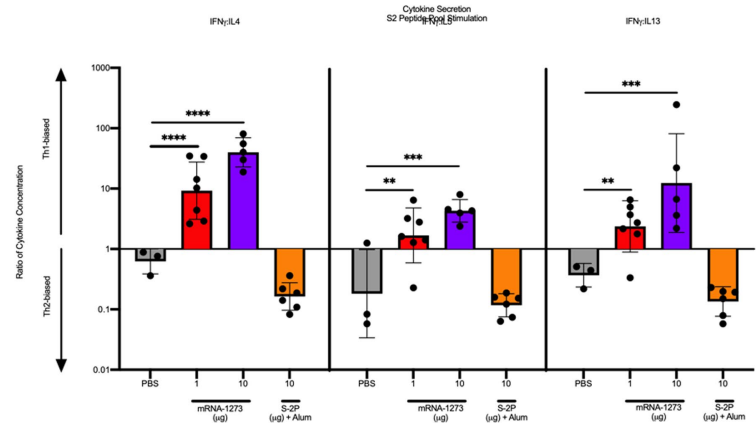

Extended Data Fig. 8 | mRNA-1273 elicits $T_{H} 1$-skewed responses compared to $S-2 P$ protein adjuvanted with alum. BALB/c mice $(n=6 /$ group) were immunized at weeks 0 and 2 weeks with 1 (red) or $10 \mu \mathrm{g}$ (purple) of mRNA-1273 or $10 \mu$ g of SARS-CoV-2S-2P protein adjuvanted with alum hydrogel (orange). Control mice were administered PBS (grey) $(n=3)$. a, b, Sera were collected 2 weeks post-boost and assessed by ELISA for SARS-CoV-2S-specific IgG1 and IgG2a. End-point titres (a) and end-point titre ratios of IgG2a to IgG1 (b) were calculated.c, d, Splenocytes were collected 4 weeks post-boost to evaluate IFN- $\gamma$, IL-4, IL-5, and IL-13 cy tokine levels secreted by T cells re-stimulated with S1 (c) and S2 (d) peptide pools, measured by Luminex. In b, immunogens were compared by two-sided Mann-Whitney $U$-test. In c, d, for cytokines, all comparisons were compared to PBS control mice by Kruskal-Wallis ANOVA with Dunn's multiple comparisons test. ${ }^{*} P<0.05,{ }^{* *} P<0.01,{ }^{* * *} P<0.001$, ${ }^{* * * *} P<0.0001$. Data are presented as GMT \pm geometric s.d. (a) or mean \pm s.d. (b-d). Dotted line represents assay limit of detection. 
a

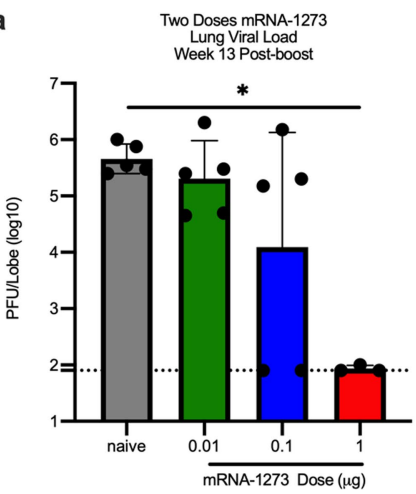

Extended Data Fig. 9 | mRNA-1273 protects mice from upper-and lower-airway SARS-CoV-2 infection, 13 weeks post-boost. a, b, BALB/cJ mice were immunized at weeks 0 and 3 with 0.01 (green), 0.1 (blue), or $1 \mu \mathrm{g}$ (red) of mRNA-1273. Age-matched naive mice (grey) served as controls. Thirteen weeks post-boost, mice were challenged with mouse-adapted SARS-CoV-2. Two days

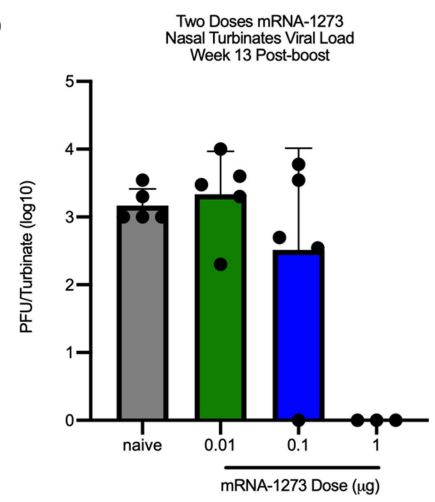

post-challenge, at peak viral load, mouse lungs (a) and nasal turbinates (b) were collected from 5 mice per group ( 3 mice for the $1 \mu$ g group) for analysis of viral titres. All dose levels were compared by Kruskal-Wallis ANOVA with Dunn's multiple comparisons test. ${ }^{*} P<0.05$. Data are presented as GMT \pm geometric s.d. Dotted line represents assay limit of detection. 

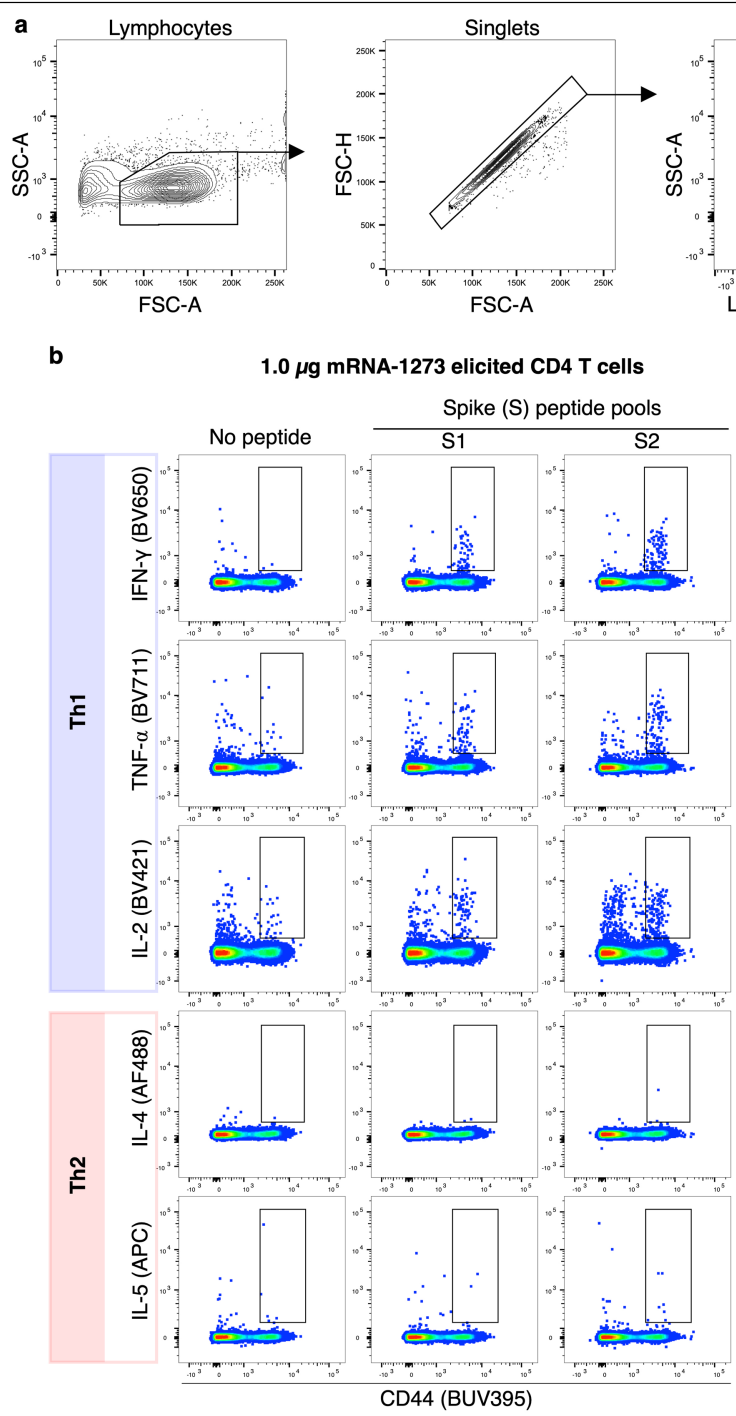

d

$1.0 \mu \mathrm{g}$ mRNA-1273 elicited CD8 T cells

Spike (S) peptide pools

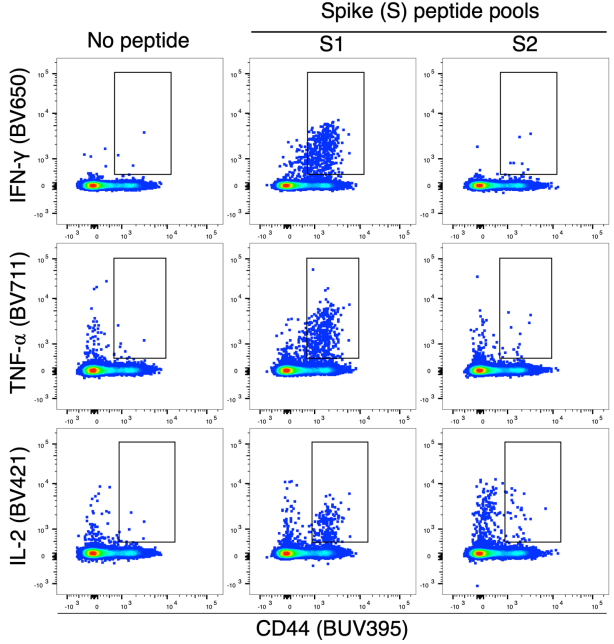

Extended Data Fig. 10 | Flow cytometry panel to quantify SARS-CoV-2 S-specific T cells in mice. a, Related to Fig. $3 \mathrm{~d}-\mathrm{g}$, a hierarchical gating strategy was used to unambiguously identify single, viable $\mathrm{CD} 4^{+}$and $\mathrm{CD} 8^{+} \mathrm{T}$ cells. b-e, Gating summary of SARS-CoV-2S-specific (b,c) $\mathrm{CD}^{+}$and $(\mathbf{d}, \mathbf{e}) \mathrm{CD} 8^{+} \mathrm{T}$ cells elicited by 0.01 and $1 \mu \mathrm{g}$ mRNA-1273 immunization. Antigen-specific T cell
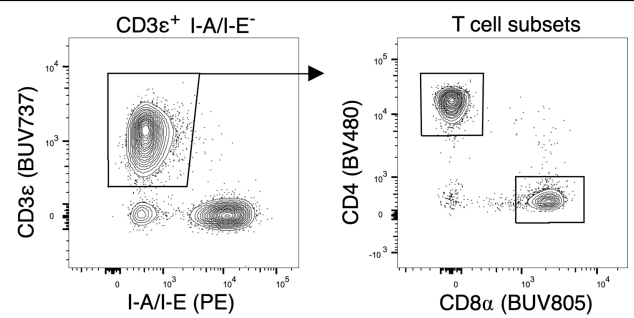

C

$0.01 \mu \mathrm{g}$ mRNA-1273 elicited CD4 T cells

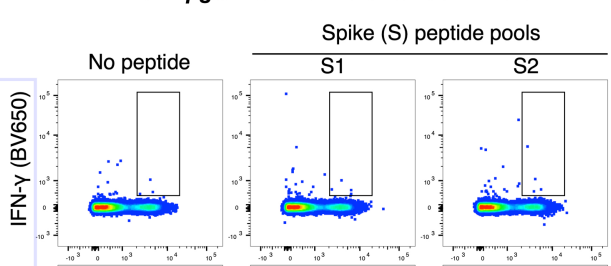

$\overline{5}$
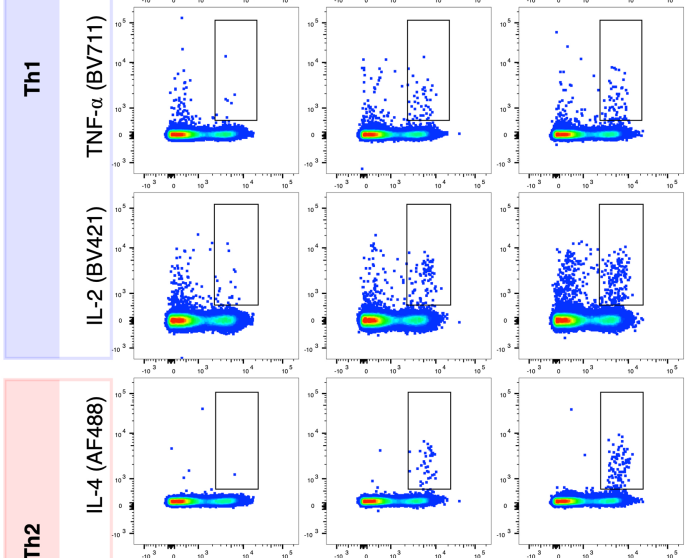

กิ

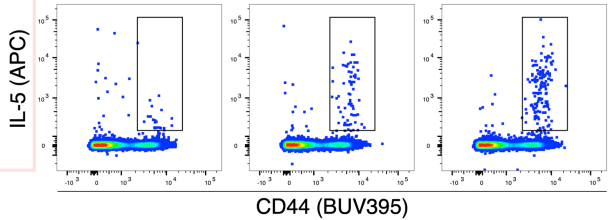

$0.01 \mu$ m mRA-1273 elicited CD8 T cells

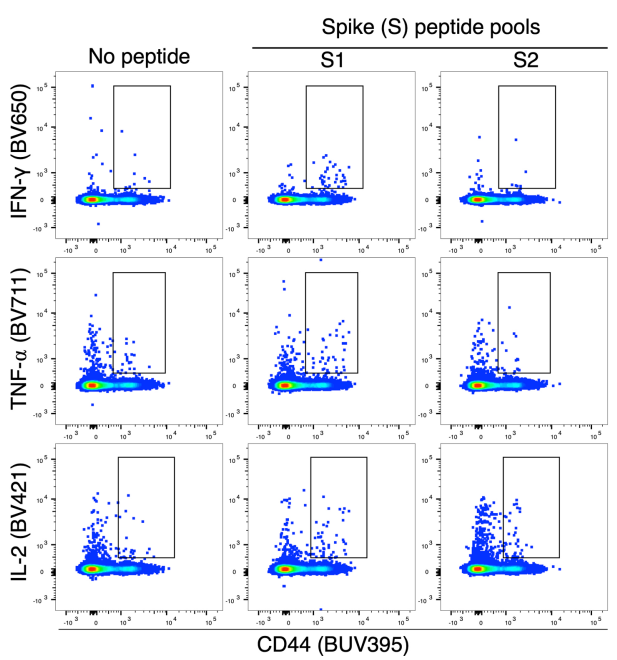

responses following peptide pool re-stimulation were defined as CD $44^{\mathrm{hi}} /$ cytokine ${ }^{+}$. Concatenated files shown were generated using the same number of randomly selected events from each animal across the different stimulation conditions using FlowJo sof tware, v.10.6.2. 


\section{Reporting Summary}

Nature Research wishes to improve the reproducibility of the work that we publish. This form provides structure for consistency and transparency in reporting. For further information on Nature Research policies, see our Editorial Policies and the Editorial Policy Checklist.

\section{Statistics}

For all statistical analyses, confirm that the following items are present in the figure legend, table legend, main text, or Methods section.

$\mathrm{n} / \mathrm{a} \mid$ Confirmed

$\square$ The exact sample size $(n)$ for each experimental group/condition, given as a discrete number and unit of measurement

$\square$ A statement on whether measurements were taken from distinct samples or whether the same sample was measured repeatedly

$\varnothing$ The statistical test(s) used AND whether they are one- or two-sided

Only common tests should be described solely by name; describe more complex techniques in the Methods section.

Х $\square$ A description of all covariates tested

$\square \bigotimes$ A description of any assumptions or corrections, such as tests of normality and adjustment for multiple comparisons

$\square$ A full description of the statistical parameters including central tendency (e.g. means) or other basic estimates (e.g. regression coefficient)

AND variation (e.g. standard deviation) or associated estimates of uncertainty (e.g. confidence intervals)

$\varnothing$ For null hypothesis testing, the test statistic (e.g. $F, t, r$ ) with confidence intervals, effect sizes, degrees of freedom and $P$ value noted

Give P values as exact values whenever suitable.

$\bigotimes \square$ For Bayesian analysis, information on the choice of priors and Markov chain Monte Carlo settings

$\bigotimes \square$ For hierarchical and complex designs, identification of the appropriate level for tests and full reporting of outcomes

$\bigotimes \square$ Estimates of effect sizes (e.g. Cohen's $d$, Pearson's $r$ ), indicating how they were calculated

Our web collection on statistics for biologists contains articles on many of the points above.

\section{Software and code}

Policy information about availability of computer code

Data collection No software used

Data analysis R v4.0.0, Prism v8 (Graph Pad), FlowJo v10.6.2

For manuscripts utilizing custom algorithms or software that are central to the research but not yet described in published literature, software must be made available to editors and reviewers. We strongly encourage code deposition in a community repository (e.g. GitHub). See the Nature Research guidelines for submitting code \& software for further information.

\section{Data}

Policy information about availability of data

All manuscripts must include a data availability statement. This statement should provide the following information, where applicable:

- Accession codes, unique identifiers, or web links for publicly available datasets

- A list of figures that have associated raw data

- A description of any restrictions on data availability

The authors declare that the data supporting the findings of this study are available within the paper and it's supplementary information files. 


\section{Field-specific reporting}

Please select the one below that is the best fit for your research. If you are not sure, read the appropriate sections before making your selection. $\bigotimes$ Life sciences $\quad \square$ Behavioural \& social sciences $\quad \square$ Ecological, evolutionary \& environmental sciences

For a reference copy of the document with all sections, see nature.com/documents/nr-reporting-summary-flat.pdf

\section{Life sciences study design}

All studies must disclose on these points even when the disclosure is negative.

Sample size Sample size for animal experiments was determined based on criteria set by institutional ACUC.

Data exclusions No data were excluded.

Replication Animal studies were completed once. All immunoassay testing was completed in duplicate or triplicate with 1 replicate, unless otherwise stated.

Randomization Allocation of animals was not random.

Blinding Blinding was not completed as assays were completed by the same team that immunized animals.

\section{Reporting for specific materials, systems and methods}

We require information from authors about some types of materials, experimental systems and methods used in many studies. Here, indicate whether each material, system or method listed is relevant to your study. If you are not sure if a list item applies to your research, read the appropriate section before selecting a response.

Materials \& experimental systems Methods

n/a Involved in the study

$\square$ Antibodies

$\square \bigotimes$ Eukaryotic cell lines

\ $\square$ Palaeontology and archaeology

$\mathrm{n} / \mathrm{a}$ Involved in the study

$\square \bigotimes$ Animals and other organisms

\ $\square$ ChIP-seq

$\searrow \square$ Human research participants

$\bigotimes \square$ Clinical data

$\bigotimes \square$ Dual use research of concern

\section{Antibodies}

Antibodies used

CR3022 (made in house, citation below) | For ICS, a surface stain cocktail containing the following antibodies: I-A/I-E PE (BD, cat. 557000, clone M5/114.15.2, 1/2500), CD8a BUV805 (BD, cat. 612898, clone 53-6.7, 1/80), CD44 BUV395 (BD, cat. 740215, clone IM7, 1/800), CD62L BV605 (Biolegend, cat. 104418, clone MEL-14, 1/5000), and CD4 BV480 (BD, cat. 565634, clone RM4-5, 1/500)

Validation

Jan ter Meulen, J. et al. Human Monoclonal Antibody Combination against SARS Coronavirus: Synergy and Coverage of Escape Mutants. PLOS Medicine 3, e237, doi:10.1371/journal.pmed.0030237 (2006).

\section{Eukaryotic cell lines}

Policy information about cell lines

Cell line source(s)

Expi293 (ThermoFisher), HEK293T/17 (ATCC \#CRL-11268), Vero E6 (ATCC), Huh7.5 cells (provided by Deborah R. Taylor, US Food and Drug Administration), ACE-2-expressing 293T (ATCC) cells (provided by Michael Farzan, Scripps Research Institute). Huh7.5 cells are a derivative of Huh7 cells (ATCC).

Authentication

Cell lines were not authenticated.

Mycoplasma contamination

All cells tested negative for mycoplasma.

Commonly misidentified lines (See ICLAC register)

No commonly misidentified cell lines are in this study. 
Animals and other organisms

Policy information about studies involving animals; ARRIVE guidelines recommended for reporting animal research

Laboratory animals

6-8-week-old female BALB/c (Charles River), BALB/CJ, C57BL/6J, or B6C3F1/J mice (Jackson Laboratory) | 16-20-week-old male and female $288 / 330+/+$ mice

Wild animals

There were no wild animals used in this study

Field-collected samples There were no field-collected samples.

Ethics oversight

Animal experiments were carried out in compliance with all pertinent US National Institutes of Health regulations and approval from the Animal Care and Use Committee of the Vaccine Research Center, Moderna Inc., or University of North Carolina at Chapel Hill.

Note that full information on the approval of the study protocol must also be provided in the manuscript.

\section{Flow Cytometry}

Plots

Confirm that:

$\triangle$ The axis labels state the marker and fluorochrome used (e.g. CD4-FITC).

\The axis scales are clearly visible. Include numbers along axes only for bottom left plot of group (a 'group' is an analysis of identical markers).

$\bigotimes$ All plots are contour plots with outliers or pseudocolor plots.

$\bigotimes$ A numerical value for number of cells or percentage (with statistics) is provided.

\section{Methodology}

Sample preparation

Instrument

Software

Cell population abundance

Gating strategy
Mononuclear single cell suspensions from whole mouse spleens were generated using a gentleMACS tissue dissociator (Miltenyi Biotec) followed by $70 \mu \mathrm{m}$ filtration and density gradient centrifugation using Fico/Lite-LM medium (Atlanta Biologicals). Cells from each mouse were resuspended in R10 media (RPMI 1640 supplemented with Pen-Strep antibiotic, $10 \% \mathrm{HI}-\mathrm{FBS}$, Glutamax, and HEPES) and incubated for $6 \mathrm{hr}$ at $37^{\circ} \mathrm{C}$ with protein transport inhibitor cocktail (eBioscience) under three conditions: no peptide stimulation, and stimulation with two spike peptide pools (JPT product PM-WCPV-S-1). Peptide pools were used at a final concentration of $2 \mu \mathrm{g} / \mathrm{mL}$ each peptide. Cells from each group were pooled for stimulation with cell stimulation cocktail (eBioscience) as a positive control. Following stimulation, cells were washed with PBS prior to staining with LIVE/DEAD Fixable Blue Dead Cell Stain (Invitrogen) for $20 \mathrm{~min}$ at RT. Cells were then washed in FC buffer (PBS supplemented with 2\% HI-FBS and 0.05\% NaN3) and resuspended in BD Fc Block (clone 2.4G2) for 5 min at RT prior to staining with a surface stain cocktail containing the following antibodies purchased from $\mathrm{BD}$ and Biolegend: I-A/I-E (M5/114.15.2) PE, CD8a (53-6.7) BUV805, CD44 (IM7) BUV395, CD62L (MEL-14) BV605, and CD4 (RM4-5) BV480 in brilliant stain buffer (BD). After $15 \mathrm{~min}$, cells were washed with FC buffer then fixed and permeabilized using the BD Cytofix/Cytoperm fixation/permeabilization solution kit according to manufacturer instructions. Cells were washed in perm/wash solution and stained with Fc Block (5 min at RT), followed by intracellular staining $\left(30 \mathrm{~min}\right.$ at $\left.4^{\circ} \mathrm{C}\right)$ using a cocktail of the following antibodies purchased from BD, Biolegend, or eBioscience: CD3e (17A2) BUV737, IFN- $\gamma$ (XMG1.2) BV650, TNF- $\alpha$ (MP6-XT22) BV711, IL-2 (JES6-5H4) BV421, IL-4 (11B11) Alexa Fluor 488, and IL-5 (TRFK5) APC in 1x perm/wash diluted with brilliant stain buffer. Finally, cells were washed in perm/wash solution and resuspended in 0.5\% PFA-FC stain buffer prior to running on a Symphony A5 flow cytometer (BD). Analysis was performed using FlowJo software, version 10.6 .2 according to the gating strategy outlined in Extended Data Figure 9. Background cytokine expression in the no peptide condition was subtracted from that measured in the S1 and S2 peptide pools for each individual mouse.

Symphony A5 flow cytometer (BD)

FlowJo software, version 10.6.2

Concatenated files shown were generated using the same number of randomly selected events from each animal across the different stimulation conditions.

Extended Data Fig. 10 shows a hierarchical gating strategy was used to unambiguously identify single, viable CD4+ and CD8+ T cells. Gating summary of SARS-CoV-2 S-specific CD4 (b-c) and CD8 (d-e) T cells. Antigen-specific T cell responses following peptide pool re-stimulation were defined as CD44hi/cytokine+.

Tick this box to confirm that a figure exemplifying the gating strategy is provided in the Supplementary Information. 